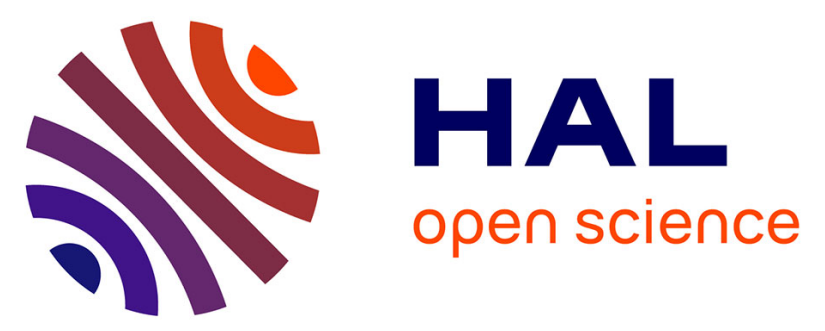

\title{
Timescales for electron quasi-linear diffusion by parallel and oblique lower-band chorus waves
}

\author{
D Mourenas, A.V. Artemyev, J.-F Ripoll, O. V. Agapitov, V.V.
}

Krasnoselskikh

\section{- To cite this version:}

D Mourenas, A.V. Artemyev, J.-F Ripoll, O. V. Agapitov, V.V. Krasnoselskikh. Timescales for electron quasi-linear diffusion by parallel and oblique lower-band chorus waves. Journal of Geophysical Research Space Physics, 2012, 117, A06234 (17 p.). 10.1029/2012JA017717 . insu-01179755

HAL Id: insu-01179755

https://hal-insu.archives-ouvertes.fr/insu-01179755

Submitted on 23 Jul 2015

HAL is a multi-disciplinary open access archive for the deposit and dissemination of scientific research documents, whether they are published or not. The documents may come from teaching and research institutions in France or abroad, or from public or private research centers.
L'archive ouverte pluridisciplinaire HAL, est destinée au dépôt et à la diffusion de documents scientifiques de niveau recherche, publiés ou non, émanant des établissements d'enseignement et de recherche français ou étrangers, des laboratoires publics ou privés. 


\title{
Timescales for electron quasi-linear diffusion by parallel and oblique lower-band chorus waves
}

\author{
D. Mourenas, ${ }^{1}$ A. V. Artemyev, ${ }^{2,3}$ J.-F. Ripoll, ${ }^{1}$ O. V. Agapitov, ${ }^{2,4,5}$ \\ and V. V. Krasnoselskikh ${ }^{2}$ \\ Received 12 March 2012; revised 25 April 2012; accepted 21 May 2012; published 30 June 2012.
}

[1] The loss of relativistic electrons from the Earth's radiation belts can be described in terms of the quasi-linear pitch angle diffusion by cyclotron-resonant waves, provided that their frequency spectrum is broad enough. Chorus waves at large wave-normal angles with respect to the magnetic field are often present in CLUSTER and THEMIS measurements in the outer belt at moderate to high latitudes. An approximate analytical formulation of diffusion coefficients has been derived in the low-frequency limit, leading to a simplified analytical expression of diffusion coefficients and lifetimes for energetic trapped electrons. Large values of the wave-normal angles between the Gendrin and resonance angles are shown to induce important increases in diffusion, thereby strongly reducing the particle lifetimes (by almost two orders of magnitude). The analytical diffusion coefficients and lifetimes obtained here are found to be in a good agreement with full numerical calculations based on CLUSTER chorus waves measurements in the outer belt for electron energies ranging from $100 \mathrm{keV}$ to $2 \mathrm{MeV}$. Such very oblique chorus waves could contribute to a predominantly perpendicular anisotropy of the global equatorial electron population on the dayside and to a relative isotropization at low energy under disturbed conditions. It is also suggested that they might play a significant role in pulsating auroras.

Citation: Mourenas, D., A. V. Artemyev, J.-F. Ripoll, O. V. Agapitov, and V. V. Krasnoselskikh (2012), Timescales for electron quasi-linear diffusion by parallel and oblique lower-band chorus waves, J. Geophys. Res., 117, A06234, doi:10.1029/2012JA017717.

\section{Introduction}

[2] Understanding the dynamics of the Van Allen radiation belts is of both theoretical and practical importance. Intense fluxes of relativistic $(\mathrm{MeV})$ electrons occurring during periods of important geomagnetic disturbances can damage satellites electronic components, which has spurred increasing interest in the field of "space weather" forecasting [e.g., see Barker et al., 2005]. Different competing acceleration and loss mechanisms can be simultaneously at work, however, making global belt modeling a very challenging task. Multidimensional codes have been developed to simulate the dynamics of the radiation belts, some of them with a special focus on the outer belt where radial diffusion is believed to be of major importance [Bourdarie et al., 1996; Barker et al., 2005; Ukhorskiy et al., 2005; Shprits et al., 2008; Varotsou et al., 2008; Xiao et al., 2009; Fok et al., 2011]. The

\footnotetext{
${ }^{1} \mathrm{CEA}, \mathrm{DAM}, \mathrm{DIF}$, Arpajon, France.

${ }^{2}$ LPC2E/CNRS-University of Orleans, Orleans, France.

${ }^{3}$ Space Research Institute, RAS, Moscow, Russia.

${ }^{4}$ Le Studium, Institute for Advanced Studies, Orleans, France.

${ }^{5}$ Astronomy and Space Physics Department, National Taras Shevchenko University of Kiev, Kiev, Ukraine.

Corresponding author: D. Mourenas, CEA, DAM, DIF, F-91297 Arpajon, France. (didier.mourenas@cea.fr)

(C)2012. American Geophysical Union. All Rights Reserved. 0148-0227/12/2012JA017717
}

outer radiation belt exhibits a very high variability, due to rapidly evolving natural conditions such as convection and geomagnetic activity levels in response to solar wind changes [Meredith et al., 2001; Lyons et al., 2005]. In particular, an important population of plasma sheet low-energy electrons $(E \sim 10-100 \mathrm{keV})$ are injected near midnight in the outer belt during storms or substorms. Drifting from midnight through dawn to noon, such low-energy electrons are believed to excite lower-band chorus waves near the equator through thermal anisotropy or loss cone instability at small pitch angles, the generated quasi-parallel whistler waves in turn accelerating some electrons to relativistic energies at large pitch angles in the night sector and scattering particles into the loss cone at small pitch angles on the dawnside, finally leading to precipitations in the atmosphere and pulsating auroras [Horne et al., 2005; Li et al., 2007; Summers et al., 2007b; Ni et al., 2008; Nishimura et al., 2011]. The inferred timescales for precipitation range from hours to days during periods of storm-enhanced convection [Horne et al., 2005; Li et al., 2007]. But these previous studies have focused on parallel or moderately oblique waves dominant near the equator [Shprits et al., 2007; Summers et al., 2007b; Ni et al., 2008; Shprits and $N i, 2009$ ], while recent CLUSTER measurements have demonstrated that very oblique chorus waves become statistically predominant at moderate to high latitudes $>15^{\circ}$, especially on the dayside where they reach high amplitudes [Agapitov et al., 2011; Artemyev et al., 2012]. In order to 


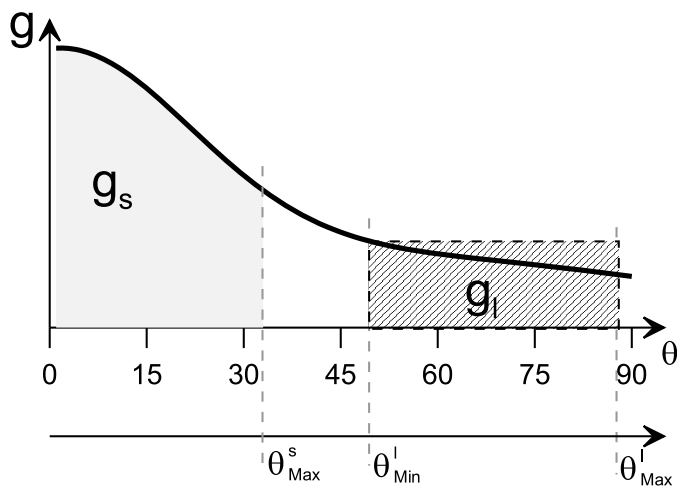

Figure 1. Schematic view of $g(\theta)$ distribution.

properly model the outer belt dynamic evolution, it is therefore important to take into account the dependence of electronchorus wave interaction on latitude and wave-normal angle [Glauert and Horne, 2005; Shprits and Ni, 2009] and to understand its impact on timescales as a function of electron energy, plasma density and $L$-shell.

[3] Chorus waves are intense whistler mode electromagnetic waves of time-varying frequencies occurring outside the plasmapause in two distinct frequency bands [Tsurutani and Smith, 1977]. Here, we shall focus on the lower-band chorus waves in the low-frequency limit, i.e. $\Omega_{c i} \ll \omega<\Omega_{c} / 2$ and $\Omega_{p e}^{2} \gg \omega \Omega_{c}$ where $\omega$ is the wave frequency, and $\Omega_{c i}, \Omega_{c}$ and $\Omega_{p e}$ are the local ion gyrofrequency, electron gyrofrequency, and plasma frequency, respectively. Occurring mainly in the dawn sector, they represent the most intense chorus measured in disturbed conditions and are probably generated close to the geomagnetic equator by anisotropic injected electrons [Tsurutani and Smith, 1977; Goldstein and Tsurutani, 1984; Muto et al., 1987; Hattori et al., 1991; Trakhtengerts, 1999; Pokhotelov et al., 2008; Santolik et al., 2009; Agapitov et al., 2010, 2011; Haque et al., 2011].

[4] Pitch angle scattering by resonant interaction with whistler waves has long been known as one of the main loss processes of trapped electrons [Andronov and Trakhtengerts, 1964; Kennel and Petschek, 1966]. Quasi-linear theory can be used to quantify such losses [Lyons et al., 1971, 1972; Lyons, 1974] in the limits of moderate amplitude broadband waves or for ensemble averages of narrowband waves of space-varying frequencies, such that the trapped particles displacements can be considered stochastic [Summers et al., 2007a; Tao et al., 2011]. However, multidimensional simulations require a very large number of diffusion coefficient evaluations. In order to calculate them as quickly as possible, Summers [2005] and Albert [2007] have proposed different useful approximations based on the selection of a given wave normal angle $\theta$ representative of the average over the whole distribution. Shprits et al. [2006] showed in particular that for high-energy electrons $(E>100 \mathrm{keV})$, the particle lifetimes can be estimated from the diffusion coefficients calculated for first cyclotron resonance with parallel-propagating chorus waves when $\theta<\pi / 4$.

[5] In the present paper, analytical expressions for the diffusion coefficients and lifetimes are derived after performing both an analytical wave-normal averaging and a rough bounce-average, following a method introduced by Mourenas and Ripoll [2012]. However, the latter work focused on moderately oblique very low frequency hiss waves, appropriate for the inner radiation belt [Meredith et al., 2009]. Here, higher-order terms in $\gamma \omega / \Omega_{c}$ and $\omega /\left(\Omega_{c} \cos \theta\right)$ are retained to allow for the treatment of energetic $(\sim \mathrm{MeV}$, i.e. relativistic factor $\gamma \sim 3$ ) electron interaction with very oblique lowerband chorus emissions. In addition, the distribution in wavenormal angle $g(\theta)$ is written as a sum of two parts corresponding to small and large $\theta$, allowing us to account for the large variance and mean value of the measured distribution of chorus waves in the outer belt [Haque et al., 2010; Agapitov et al., 2011; Li et al., 2011]. Approximate scaling laws of electron lifetimes as a function of energy, ambient density and $L$-shell are also derived in section 2 , and the importance of electron diffusion by very oblique chorus waves is stressed. In section 3, the analytical model is compared to electron loss timescales [Shprits et al., 2007] obtained from numerical computations of the diffusion coefficients using Summers [2005] parallel propagation approximation and to full numerical calculations including a realistic wave-normal distribution. The analytical estimates are found to be in good agreement with the full numerical results and help to provide a better understanding of the important reduction of timescales obtained when taking into account very oblique waves. Some potential physical implications concerning the dynamics of the outer radiation belt are also outlined.

\section{Analytical Estimates of Diffusion Coefficients}

[6] Observation of oblique whistler wave propagation [Haque et al., 2010; Agapitov et al., 2011; Li et al., 2011] can be taken into account for estimates of diffusion rates by introducing a double $\theta$-distribution $g(\theta)$. Instead of considering only a narrow distribution around $\theta=0$, one should treat a distribution containing a "heavy" tail (see scheme in Figure 1). This non-Gaussian tail includes $\theta$ around the resonance angle and, as a result, can be responsible for the intensification of high order resonances in the electron interaction with whistler waves. In this paper we estimate the role of this tail by separating the distribution dependence upon the wave-normal angle $\theta$ into two parts $g(\theta)=g_{s}(\theta)+g_{l}(\theta)$. The small- $\theta$ part $g_{s}(\theta)$ is such that its maximum occurs at $\theta_{m}^{s} \approx 0$ with lower and upper cutoffs at $\theta_{\text {Min }}^{s} \approx 0$ and $\theta_{\text {Max }}^{s}$. The large- $\theta$ part is taken as a step-function $g_{l}(\theta)$ equal to a constant between its lower and upper cutoffs ( $\theta_{\mathrm{Min}}^{l}$ and $\left.\theta_{\mathrm{Max}}^{l}\right)$ being zero otherwise. Below we show that final estimates do not depend on the actual form of $g_{l}(\theta)$. We further assume that $\theta_{\mathrm{Max}}^{s} \leq \theta_{\mathrm{Min}}^{l}$.

[7] The local pitch angle quasi-linear diffusion coefficient D of Lyons [1974] has been conveniently rewritten by Albert [2007] under the form of a weighted-average between $\theta_{\text {Min }}$ and $\theta_{\text {Max }}$, so that $D \sim\langle D\rangle_{\theta}$. Moreover, Albert [2007] emphasized that this weighted average of relatively constant factors is expected to be rather insensitive to the exact form of the weighting function. Therefore, the averaged function may be taken at a "carefully chosen point" using the Mean Value Approximation (MVA) [Albert, 2007, 2008], yielding to $D \sim D\left(\theta_{0}\right)$. The parallel propagation approximation (PPA) derived earlier [Summers, 2005] essentially consists in taking $\theta_{0}=0$.

[8] Although the present approach starts from the above mentioned weighted-average formulation, it is performed slightly differently to keep at least some of the variations of the averaged function with $\theta$. As discussed in a previous 
paper [Mourenas and Ripoll, 2012], the diffusion coefficient for each harmonic can be estimated near the maximum of each cyclotron resonance $n$, corresponding to a Bessel function maximum. However, we shall focus here on lifetimes. Albert and Shprits [2009] have demonstrated that lifetimes are defined by the minima of the total diffusion coefficient multiplied by tangent of pitch angle $\alpha$. Thus, for the small- $\theta$ part we shall neglect all the resonances except for the cyclotron ones at small pitch angles and the Landau resonance at large pitch angles, which should roughly define the actual lifetimes, since the diffusion coefficient is usually the smallest either near the loss cone angle $\alpha_{L C}$ or, at large pitch angles, between the Landau and first cyclotron resonances [Shprits et al., 2006, 2007; Albert and Shprits, 2009; Mourenas and Ripoll, 2012]. For large- $\theta$ waves, it is the moderate to large pitch angles region which will be more important. Here, the situation is substantially more complicated and many resonances contribute to diffusion.

\subsection{Generalities}

[9] The local pitch angle quasi-linear diffusion coefficient $D$ of Lyons [1974] (with dimensions of $\mathrm{s}^{-1}$ ) has been rewritten by Albert [2005, 2007] as

$$
\begin{aligned}
& D=\frac{D^{\alpha \alpha}}{p^{2}}=\frac{\Omega_{c}}{\gamma^{2}} \frac{B_{\text {wave }}^{2}}{B^{2}} \sum_{n=-\infty}^{+\infty} \sum_{\omega} D_{n}^{\alpha \alpha} \\
& D_{n}^{\alpha \alpha}=\int_{\theta_{\min }}^{\theta_{\max }} \sin (\theta) d \theta \Delta_{n} G_{\omega} G_{\theta}
\end{aligned}
$$

with

$$
\begin{gathered}
\Delta_{n}=\frac{\pi \Phi_{n}^{2}}{2 \cos \theta\left|v_{\|} / c\right|^{3}} \frac{\left|\sin ^{2}(\alpha)+n \Omega_{c} / \gamma \omega\right|^{2}}{\left|1-\left(\partial \omega / \partial k_{\|}\right)_{\theta} / v_{\|}\right|} \\
G_{\omega}=\frac{\Omega_{c} B_{s}^{2}(\omega)}{\int_{\omega_{L}}^{\omega_{U}} B_{s}^{2}\left(\omega^{\prime}\right) d \omega^{\prime}}, G_{\theta}(\omega, \theta)=\frac{g(\theta)}{N(\omega, \theta)} \\
N(\omega, \theta)=\int_{\theta_{\min }}^{\theta_{\max }} d \theta^{\prime} \sin \left(\theta^{\prime}\right) \Gamma g\left(\theta^{\prime}\right) \\
\Gamma\left(\theta^{\prime}, \omega(\theta)\right)=\mu^{2}|\mu+\omega \partial \mu / \partial \omega|
\end{gathered}
$$

where $\mu(\omega, \theta)=k c / \omega$ is the wave refractive index, $B$ is the local magnetic field amplitude, $\Phi_{n}^{2}$ accounts for the relationship between the electric and magnetic field components of the wave and it is given in equation (9) of Lyons [1974]; it contains Bessel functions $\mathrm{J}_{n \pm 1}$ with argument $x=\left(\omega \gamma / \Omega_{c}+n\right) \tan \alpha$ $\tan \theta$, which can be approximated by $x \sim n \tan \alpha \tan \theta$ at low frequency when $\omega \gamma / \Omega_{c} \ll 1 \leq n$, where $\gamma$ is the relativistic factor. $B_{s}^{2}(\omega)=\exp \left(-\left(\omega-\omega_{m}\right)^{2} / \Delta \omega^{2}\right)$ is the wave spectral density, with a half-width $\Delta \omega<\omega_{m} / 2$ and lower and upper cut-offs at $\omega_{L} \sim \omega_{m} / 2$ and $\omega_{U} \sim 1.5 \omega_{m}$. In equation (1), $G_{\theta}(\omega, \theta)$ and $D_{n}^{\alpha \alpha}$ are both evaluated at the resonant frequency $\omega$ corresponding to a $\theta$, a harmonic number $n$, and a pitch angle $\alpha$ as determined from the cyclotron resonance condition

$$
\omega+n \Omega_{c} / \gamma=k v \cos \theta \cos \alpha
$$

where $v$ denotes the electron velocity. There may be several roots of $\omega$, hence the sum over $\omega$ in equation (1). An important point is that for each $n, D_{n}^{\alpha \alpha}$ and $G_{\theta}(\omega, \theta)$ depend only on $\theta$. Cyclotron resonances occur during the bounce motion of electrons from the equator to their mirror point, and bounce averaging is described in the classic paper of Lyons et al. [1972].

\subsection{Estimates of Contributing Cyclotron Resonances}

[10] In the low-frequency limit $\omega / \Omega_{c} \ll \min \left(\Omega_{p e}^{2} / \Omega_{c}^{2}, 1\right)$, the dispersion relation is $\mu^{2}=\Omega_{p e}^{2} /\left(\omega_{m} \Omega_{c}|\cos \theta|-\omega_{m}^{2}\right)$ [Helliwell, 1965] where the resonance maximum is hereafter assumed to always occur at $\omega \sim \omega_{m}$. The first-order approximation $\omega \sim \omega_{m}$ introduced in Mourenas and Ripoll [2012] can be justified by: (1) the nearly constant $\omega$ up to $\theta \sim 60^{\circ}-70^{\circ}$ for low-frequency chorus waves [Albert, 2007, 2008] using the full dispersion relation and (2) the narrow width $\Delta \omega<\omega_{m} / 2$ assumed for $B_{s}^{2}(\omega)$, implying that significant contributions to the diffusion coefficient will mainly come from frequencies close to $\omega_{m}$.

[11] In Appendix A we derive the equation for resonant value of pitch angle $\alpha_{R}$ (see equation (A1)) and we note in Appendix B that most significant contributions to diffusion at low latitudes come from the maximum of the Bessel functions of argument $x$. This condition in turn leads to a relationship between $\theta$ and $\alpha$ values (see equation (B1) in Appendix B). Subscript " $M$ " is used throughout the paper to indicate values taken at the maximum of the Bessel functions. To estimate the input of various resonances we separate the domain of $\theta$ (and $\alpha$ ) integration into two subregions: before and after the maximum of Bessel function. The use of a \pm superscript will hereafter differentiate the domains $x \geq x_{M}$ and $x \leq x_{M}$, respectively. The first part of the integral corresponds to $x \geq x_{M}$, i.e. $\tan \alpha_{R} \tan \theta>1$ or wave-normal angles $\theta$ greater than the value $\theta_{M}$ at the Bessel function maximum $\theta>\theta_{M}=\pi / 2-\alpha_{R}$. The second one concerns the $x<x_{M}\left(\right.$ or $\left.\tan \alpha_{R} \tan \theta \leq 1\right)$ domain (see Appendix B).

[12] In the limit where $\theta \sim \theta_{\text {Max }}$, the maximum number of contributing resonances is given by equation (A1) with $\cos \alpha_{R} \sim \sin \theta_{\text {Max }}$. We get

$$
N_{r} \approx \frac{p \varepsilon_{m} \sqrt{\cos \theta_{\text {Max }}} \sin \theta_{\text {Max }}}{\left|1-\frac{\omega_{m}}{\Omega_{c} \cos \theta_{\text {Max }}}\right|^{1 / 2}}+\frac{\gamma \omega_{m}}{\Omega_{c}}
$$

which is a generalization of equation (22) of Mourenas and Ripoll [2012]. Here $\varepsilon_{m}=\Omega_{p e} / \Omega_{c} \sqrt{\omega_{m} / \Omega_{c}}$ and $p$ is electron momentum normalized on $m_{e} c$ (see Appendix A). Note that equation (6) gives the total number of contributing resonances over the whole range of pitch angles. In the special cases of small equatorial pitch angles near the loss cone at small- $\theta$ and for moderate pitch angles at large- $\theta$ we can derive new estimates concerning the number of contributing resonances.

[13] For the small- $\theta$ part, we show in Appendix B that only the first cyclotron resonance $n=-1$ provides a significant contribution to the diffusion coefficient near the loss cone edge. In such a case, the $\mathrm{J}_{0}$ term is indeed dominant in $\Phi_{-1}^{2}$. As concerns the $n=+1$ resonance, it is negligible at small $\theta<\pi / 4$ as compared to the $n=-1$ one [Mourenas and Ripoll, 2012], and still small at higher $\theta$ for typical distributions such that $g_{s}\left(\theta_{g}\right) \ll g_{s}(0)$ and/or $E \leq 1 \mathrm{MeV}$, 


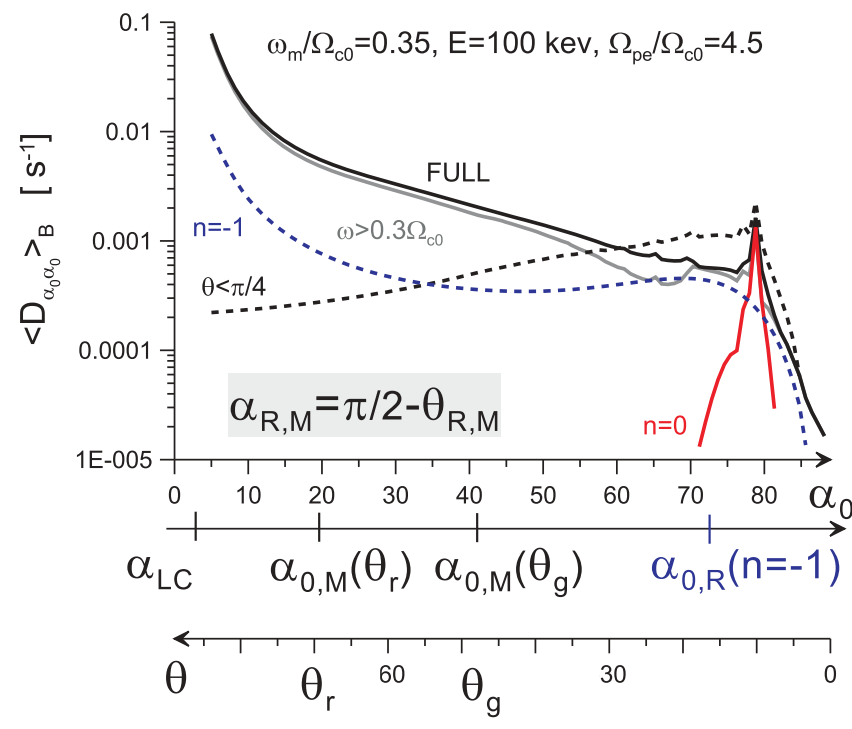

Figure 2. Bounce-averaged pitch angle diffusion coefficient as a function of equatorial pitch angle $\alpha_{0}$ for $100 \mathrm{keV}$ electrons interacting with lower-band chorus waves such that $\omega_{m}=0.35 \Omega_{c 0}$ and $\Delta \omega=0.15 \Omega_{c 0}$, with a realistic wave number angular distribution obtained from CLUSTER measurements [Agapitov et al., 2011] at $L \sim 4.5\left(\Omega_{p e} / \Omega_{c}=4.5\right)$. The full-numerical solution is plotted (solid black line) with the solutions for high-frequency waves only (grey line) and for small- $\theta\left(<45^{\circ}\right)$ part of the distribution only (dashed black line). Also shown are the $n=-1$ and $n=0$ resonances solutions (blue and red lines). The main characteristic pitch angles are displayed, as well as the corresponding wave-normal directions on the lowest axis. Description of calculations can be found in Appendix E.

where $\theta_{g}=\arccos \left(2 \omega_{m} / \Omega_{c}\right)$ is Gendrin angle. This contribution is essentially equivalent to the Parallel Propagation Approximation (PPA) proposed by Summers [2005].

[14] For the large- $\theta$ part $\theta>\max \left(\pi / 4, \theta_{g}\right)$, full numerical calculations of the bounce-averaged diffusion coefficients show that diffusion always increases very strongly toward small equatorial pitch angles (e.g., see Figure 2). This increase occurs only at high latitudes, for high values of the ratio $\Omega_{c}\left(\lambda_{R}\right) / \Omega_{c 0}$ and close to the resonance cone, where the full Bessel function expressions must be kept (here, as in the rest of the paper, the subscript " 0 " indicates that the variable value is taken at the equator). Variable $\lambda_{R}$ is the "resonant" latitude for which the resonance condition (5) is satisfied for the wave and particle considered. It can be calculated making use of adiabatic invariance $\Omega_{c}\left(\lambda_{R}\right) /$ $\Omega_{c 0}=\sin ^{2} \alpha_{R} / \sin ^{2} \alpha_{0}$. This means that the upper-bound of the resonant latitude decreases with increasing $\alpha_{0}$, so that the considered increase of diffusion toward small $\alpha_{0}$ is concentrated mainly at $\sin \alpha_{0}<1 / 3$, as in Figure 2 .

[15] Now, Albert and Shprits [2009] have demonstrated that lifetimes are mainly governed by the minima of $\langle D\rangle_{B} \tan \alpha_{0}$ (where $\langle D\rangle_{B}$ is the bounce-averaged coefficient) i.e., the minima of $\langle D\rangle_{B}$ at small pitch angles. Thus, we can safely neglect the small- $\alpha_{0}$ part of the large- $\theta$ diffusion coefficients and consider only their portion going from about $\pi / 12$ up to about $\pi / 2$, where resonance occurs at moderate latitudes $\lambda<40^{\circ}$. There and at the equator, $\theta$ at $\lambda_{R}=0$ from equation (6) may take any value ranging from the Gendrin angle $\theta_{g 0}=\arccos \left(2 \omega_{m} / \Omega_{c 0}\right)$ up to nearly the resonance angle $\theta_{r 0}=\arccos \left(\omega_{m} / \Omega_{c 0}\right)$. A rough estimate of the maximum $|n|$ for resonance at the equator at $\left(\omega_{m}, \theta_{g 0}\right)$ is simply

$$
N_{r}\left(\theta_{g 0}\right) \approx 2 \cos \alpha_{0} p \varepsilon_{m 0} \sqrt{\frac{\omega_{m}}{\Omega_{c 0}}}+\frac{\gamma \omega_{m}}{\Omega_{c 0}} .
$$

[16] In order to have resonance up to nearly $\theta_{r 0}$ (at $\cos \theta_{R} \sim 1.1 \cos \theta_{r 0}$ ), equations (7) and (A1) indicate that $\omega$ must be diminished by a factor $\sqrt{2 / 5} \sim 2 / 3$. Consequently, the number of resonances at the equator present over the full $\theta$-range from $\theta_{g 0}$ to $\theta_{r 0}$ is simply

$$
\Delta N_{r 0} \approx\left(\frac{2 \Delta \omega}{\omega_{m}}-\frac{1}{3}\right) N_{r}\left(\theta_{g 0}\right)
$$

[17] If $\Delta N_{r 0}<0$ at the equator, resonance at $\theta$ close to $\theta_{r}$ for $|n|<N_{r}\left(\theta_{g 0}\right)$ occurs only at higher latitudes. When the $\theta$-range of resonance decreases, $D$ decreases in proportion (see equation (D2) in Appendix D). The different contributions to the total diffusion coefficient are calculated in the following sections. But it is already clear from equation (6) that near the loss cone edge at $L>4\left(\alpha_{L C}<5^{\circ}\right)$, the small- $\theta$ part's most important contributions comes from very small $n$ resonances (mainly $n=-1$ ), while equations (7) and (8) show that for the large- $\theta$ part, higher- $n$ resonances contribute. This agrees well with full numerical calculations in Figure 2.

[18] According to experimental data [see Agapitov et al., 2011], $g_{s}(\theta)$ is dominant in the vicinity of the equator, while $g_{l}(\theta)$ corresponds mainly to moderate magnetic latitudes $\lambda>15^{\circ}$, where $g_{s}(\theta)$ has a relatively small amplitude. For analytical estimates we do not take into account the dependence of $g_{s}(\theta)$ on magnetic latitude $\lambda$ and assume that $g_{l}(\theta)$ bounds vary with latitude like $\theta_{r}$ with $\Omega_{c}$, in rough agreement with observations. The validity of this approach is tested by comparisons of analytical formulas with the full numerical calculations of the diffusion coefficients in section 3. In numerical calculations, we use the full $g(\theta)$ depending on $\lambda$. For comparison, we also calculate in Figure 2 the diffusion coefficient for $g(\theta)=g_{s}(\theta)$ without any dependence on $\lambda$ (such wave normal distributions are often used for calculation of diffusion coefficients [see Glauert and Horne, 2005; Horne et al., 2005; Shprits and Ni, 2009]).

\subsection{Contribution of Cyclotron Resonances}

[19] As the electron leaves the equator and moves toward its mirror point along a magnetic field line, pitch angle increases due to adiabatic invariance, allowing the resonance to be restored over some latitudes. The full diffusion coefficient is obtained by integration over the bounce motion [Lyons et al., 1972]:

$$
\langle D\rangle_{B}\left(\alpha_{0}\right)=\int_{\lambda_{\min }}^{\lambda_{\max }} \frac{D(\alpha) \cos \alpha \cos ^{7} \lambda}{T\left(\alpha_{0}\right) \cos ^{2} \alpha_{0}} d \lambda
$$

where the bounce period is written as $T\left(\alpha_{0}\right) \approx 1.38-$ $0.64 \sin ^{3 / 4} \alpha_{0}$ [Davidson, 1976]. 


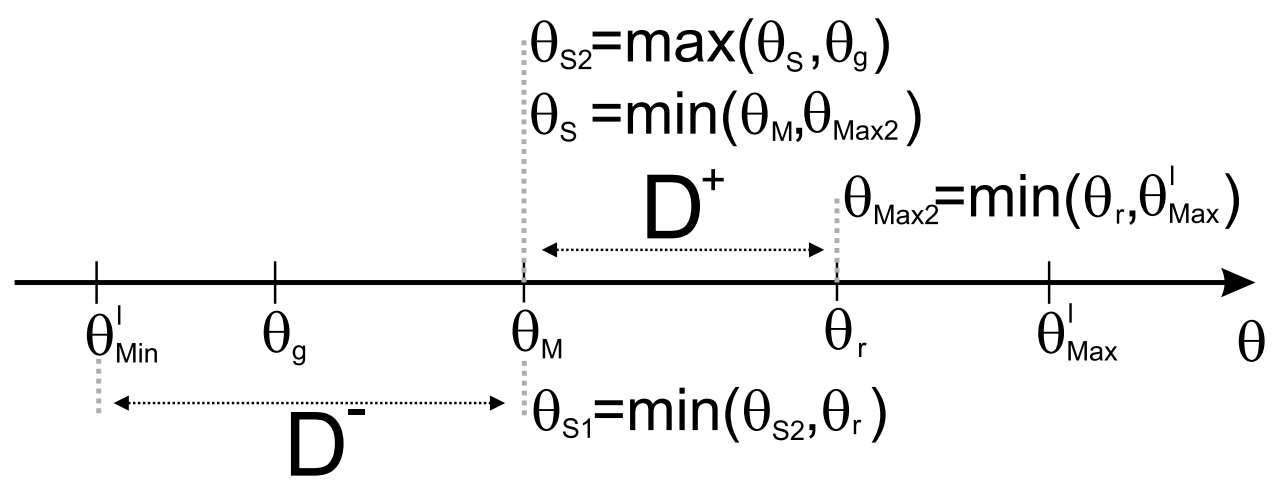

Figure 3. Schematic view of the separation of interval $\left[\theta_{\mathrm{Min}}^{l}, \theta_{\mathrm{Max}}^{l}\right]$ into two subintervals corresponding to $D^{-}$and $D^{+}$.

[20] Taking into account the weighted-average reformulation of the diffusion coefficient by Albert [2007], we estimate the bounce-averaged diffusion coefficient coming from the small- $\theta$ part in Appendix C:

$$
\begin{aligned}
\left\langle D_{-1}^{\alpha \alpha}\right\rangle_{B}^{S}\left(\alpha_{L C}\right) \approx & \frac{\pi^{3 / 2} \operatorname{erf}(1) B_{\text {wave }}^{2} \omega_{m} G_{w 0}\left(\omega_{m}\right)}{4 \gamma B_{0}^{2}\left(p \varepsilon_{m 0}\right)^{13 / 9} T\left(\alpha_{L C}\right)} s \\
& \times \frac{\Delta \lambda_{R}\left(1+3 \sin ^{2} \lambda_{R}\right)^{7 / 12}\left(1-\frac{\omega_{m}}{\Omega_{c}}\right)}{\left|\frac{\gamma \omega_{m}}{\Omega_{c}}-\frac{2 \gamma \omega_{m}^{2}}{\Omega_{c}^{2}}+1\right|\left|1-\frac{\gamma \omega_{m}}{\Omega_{c}}\right|^{4 / 9}}
\end{aligned}
$$

where $\lambda_{R}$ and $\Delta \lambda_{R}$ are resonance latitude and range of variation of resonance latitudes, respectively (see Appendix C). In equation $(10) \Omega_{c} / \Omega_{c 0} \approx\left(p \varepsilon_{m 0}\right)^{2 / 3}$ can be used for small values of $\omega_{m} / \Omega_{c 0}$ and small to moderate latitudes. The term $\lambda_{R}+\Delta \lambda_{R} / 2$ is assumed to be smaller than the upper-bound in latitude where the considered waves are present.

[21] Let us turn now to the more complicated large- $\theta$ part of $g(\theta)$, such that $\theta_{\text {Min }}^{l} \geq \max \left(\pi / 4, \theta_{g}\right)$. Important contributions to diffusion then come from resonant value $\theta_{R}$ lying between the Gendrin angle $\theta_{g}$ and the resonance angle $\theta_{r}$ (cf. Appendix B). These contributions can even be more important than those of the small- $\theta$ range, since $\Delta_{n} / \Gamma$ in equation (1) shows a singularity at the resonance angle, which can be seen in equation (A3) and which is absent at small $\theta$. The large- $\theta$ contribution to diffusion must therefore be calculated very carefully, even if it contains a much smaller amount of wave intensity than the small- $\theta$ range. Such oblique chorus waves are commonly observed in the outer belt [Hayakawa et al., 1990; Santolik et al., 2009; Tsurutani et al., 2009; Haque et al., 2010; Agapitov et al., 2011]. At the Gendrin angle, the waves remain guided along the geomagnetic field line as they propagate. However, density and magnetic field variations from moderate to high latitude usually increase the wave-normal angle until it reaches the resonance cone, potentially leading to reflection at low altitude and tending to fill the radiation belts with magnetospherically reflected whistlers [Edgar, 1976; Helliwell, 1995; Boskova et al., 1990; Shklyar and Jiřiček, 2000; Jiricek et al., 2001; Chum and Santolik, 2005]. We need to separate the interval $\left[\theta_{\mathrm{Min}}^{l}, \theta_{\mathrm{Max}}^{l}\right]$ into two subintervals $\left(\theta<\theta_{M}\right.$ and $\left.\theta>\theta_{M}\right)$ taking into account relative positions of $\theta_{g}, \theta_{r}$ (see scheme in Figure 3 ). Defining $\theta_{\text {Max2 }}=\min \left(\theta_{\text {Max }}^{l}, \theta_{r}\right), \theta_{S}=\min \left(\theta_{M}, \theta_{\text {Max } 2}\right), \theta_{S 2}=\max$
$\left(\theta_{S}, \theta_{g}\right)$, and $\theta_{S 1}=\min \left(\theta_{S 2}, \theta_{r}\right)$, the wave-normal angle integral of diffusion coefficient (see Appendix C) can be performed by splitting the integral into two parts $D^{+}$and $D^{-}$, the upper-part going from $\theta_{S 2}$ to $\theta_{\mathrm{Max} 2}$ and the lowerpart going from $\theta_{\text {Min }}^{l}$ to $\theta_{S 1}$. Roughly speaking, the upper and lower parts correspond, respectively, to the $x>x_{M}$ and $x<x_{M}$ limits in the Bessel series expansions (see equation (B2)).

[22] At pitch angles larger than $20^{\circ}$, the upper-bound of latitude for resonance is less than $40^{\circ}$ and the $D^{+}$part is expected to prevail (see Appendix B). The average latitude range $\Delta \lambda_{R}$ over which resonance exists over the whole large- $\theta$ range (from $\theta_{g}$ to $\theta_{r}$ ) can then be estimated from equations (6) and (7) where $\Omega_{c}$ is now allowed to vary with latitude. For a given $|n|$ such that $N_{r}\left(\theta_{g 0},(4 / 3) \omega_{m}-\Delta \omega\right)<$ $|n|<N_{r}\left(\theta_{g 0}, \omega_{m}+\Delta \omega\right),\left(\Omega_{c} / \Omega_{c 0}\right)^{2}$ can be increased by a factor $(1+(2 / 3)(\omega+\Delta \omega) /(\omega-\Delta \omega)) / 2$ on average. Adiabatic invariance implies that $\Omega_{c}\left(\lambda_{R}\right) / \Omega_{c 0} \sim 1+9 \lambda_{R}^{2} / 2<\sin ^{-2} \alpha_{0}$, leading to

$$
\lambda_{\max } \approx \min \left(\frac{1}{3 \sqrt{2}} \sqrt{\frac{2}{3} \frac{\omega_{m}+\Delta \omega}{\omega_{m}-\Delta \omega}-1}, \frac{\sqrt{2}}{3 \tan \alpha_{0}}\right)
$$

for the average maximum latitude of resonance. At smaller $n$-values (closer to 1 ), equation (7) is satisfied only away from the equator. There, the range of resonant latitudes $\Delta \lambda_{R}$ is slightly smaller than $\lambda_{\max }$, while $\cos \alpha_{0}$ in equation (7) should actually be replaced by $\cos \alpha_{R}$ from equation (A1) to be more exact away from the equator. From adiabatic invariance, one finds $\cos \alpha_{R} \sim\left(1-\sin ^{2} \alpha_{0} \Omega_{c} / \Omega_{c 0}\right)^{1 / 2} \sim$ $\left(\cos \alpha_{0}\right)^{\mu}$ with $\mu$ ranging from $\Omega_{c}\left(\lambda_{\max }\right) / \Omega_{c 0}$ at small $\alpha_{0}$ to $2 \Omega_{c}\left(\lambda_{\max }\right) / \Omega_{c 0}$ at large $\alpha_{0}$, yielding $\mu \sim 2$ for $\alpha_{0}=15^{\circ}$ to $70^{\circ}$. Therefore, it is reasonable to consider that roughly $2 N_{r}\left(\theta_{g 0}, \omega_{m}\right) \cos \alpha_{0}$ positive and negative resonances exist over the whole large- $\theta$ range with $\Delta \lambda_{R} \sim \lambda_{\max }$ for $\alpha_{0}>15^{\circ}$. The details of the derivation of $D^{+}$are given in Appendix D. With $\theta$-bounds taken as $\theta_{g}$ and $\theta_{r}$, this leads finally to the simplified approximate expression for the sum of resonances coefficients

$$
\left\langle D^{+}\right\rangle_{B}^{l}=\frac{\left(1+\frac{\omega_{m}^{2}}{\Omega_{c 0}^{2}}\right) B_{\text {wave }}^{2} \omega_{m} G_{\omega 0}\left(\omega_{m}\right) N_{r} \lambda_{\max }}{\gamma B_{0}^{2}\left(p \varepsilon_{m 0}\right)^{2} \cos \alpha_{0} \sin \alpha_{0}}
$$


[23] At pitch angles larger than $65^{\circ},\left\langle D^{+}\right\rangle_{B}$ has other maxima of comparable magnitude up to $\alpha_{0}^{\text {Max }}$ given by equation (B8), coming from the small- $\theta$ part contribution, and which are given by equation (30) and equation (34) of Mourenas and Ripoll [2012] for $n>0$ and $n=0$, respectively. This contribution somewhat compensates the reduction of $\lambda_{\max }$ at pitch angles larger than $60^{\circ}$ in equation (11). Moreover, the high- $\alpha_{0}$ part of $\left\langle D^{+}\right\rangle_{B}$ is much less important than the small$\alpha_{0}$ part in the lifetime integral of $1 /\left\langle D^{+}\right\rangle_{B} \tan \alpha_{0}$. From equations (7), (11) and (12), it follows that $\left\langle D^{+}\right\rangle_{B}$ varies roughly like $1 / \sin \alpha_{0}$, which is confirmed by full numerical calculations in section 3. Comparing equation (10) and equation (12), it is easy to see that large- $\theta$ diffusion can be much stronger than the small- $\theta$ diffusion, provided that a significant amount of wave energy lies inside the large- $\theta$ range.

\subsection{Total Cyclotron Diffusion Coefficient}

[24] Actually, the total $D$ is obtained in equation (1) by integration over $\omega$ of the weighted sum of expression (10) and expression for $\langle D\rangle_{B}^{l}$ (see Appendix D). The latter reads as

$$
\langle D\rangle \approx \frac{\langle D\rangle_{B}^{s} \int_{\tan \theta_{\text {Min }}^{s}}^{\tan \theta_{\text {Max }}^{s}} \Gamma(y) g(y) y d y+\langle D\rangle_{B}^{l} \int_{\tan \theta_{\text {Min }}^{l}}^{\tan \theta_{\text {Max }}^{l}} \Gamma(y) g(y) y d y}{\int_{\tan \theta_{\text {Min }}^{s}}^{\tan \theta_{\text {Max }}^{l}} \Gamma(y) g(y) y d y}
$$

where $y=\tan \theta$.

[25] Apart from $g(y)$, the weighting function $\Gamma(y) g(y) y=$ $\sqrt{1+y^{2}} y g(y)$ is very rapidly increasing with $y$ for $y=\tan \theta>1$. It means that even if the large- $\theta$ part of $g$ has a much smaller value than the small- $\theta$ part, its relative weight in equation (13) will tend to be similar due to factor $\sqrt{1+y^{2}} y>1$, so that

$$
D \approx\left(\langle D\rangle^{l}+\langle D\rangle^{s}\right) / 2
$$

is a good first-order approximation at moderate to large pitch angles for a typical proportion of wave intensity in the large$\theta$ range lying between $50 \%$ and $10 \%$ of the total intensity [Burton and Holzer, 1974; Haque et al., 2010; Agapitov et al., 2011].

\subsection{Landau Resonance}

[26] The calculation of the Landau resonance diffusion coefficient for low-frequency whistler waves has already been performed in Mourenas and Ripoll [2012], and it still applies here, at least for the small- $\theta$ part (see their equations (25) and (34)). One finds a maximum near $\cos \alpha_{M 0} \sim 11-\omega_{m} / \Omega_{c 0}{ }^{1 / 2} \gamma \omega_{m} /\left(\Omega_{c 0} p \varepsilon_{m 0}\right)$ at the equator, in agreement with simulation results, which show that the small- $\theta$ diffusion generally dominates (cf. Figure 2). The bounce-averaging procedure used above applies for the Landau resonance when $\sin \alpha_{R} \sim 1 \sim \sin \alpha_{M 0}$, provided that $p>\gamma \omega_{m} /\left(\Omega_{c 0} \varepsilon_{m 0}\right)$ from the resonance condition: it corresponds to relatively high energy electrons $E>10 \mathrm{keV}$ typically for chorus waves, in agreement with numerical results from Shprits and $\mathrm{Ni}$ [2009]. However, comparing Landau and cyclotron diffusion for chorus waves shows that Landau diffusion is similar to or smaller than cyclotron diffusion at high energies $E>300 \mathrm{keV}$. It can, thus, be neglected to estimate trapped electron timescales for $E>300 \mathrm{keV}$ [Albert and Shprits, 2009]. At lower energy and small $\omega_{m} / \Omega_{c 0}$, it can be included from the Landau part in equation (36) of Mourenas and Ripoll [2012]. This is indeed confirmed by different numerical simulations [Shprits et al., 2006; Shprits and Ni, 2009].

\subsection{Estimates of Trapped Electrons Timescales}

[27] Albert and Shprits [2009] have derived a simplified but very useful estimate of electron lifetime $\tau$, which can be used together with our analytical approximate expressions for $D$ :

$$
\tau \approx \sigma \int_{\alpha_{L C}}^{\pi / 2} \frac{\cos \alpha_{0}}{2\langle D\rangle_{B} \sin \alpha_{0}} d \alpha_{0} .
$$

[28] The numerical coefficient $\sigma \sim 1 / 2$ in our case allows to recover precisely numerical simulations where the minimum of $\langle D\rangle_{B}$ is close to the loss cone, or else when a minimum occurs at large pitch angles but remains moderately deep [Albert and Shprits, 2009]. It suggests that a rough estimate of lifetimes can be obtained by neglecting the parameter range wherein $\langle D\rangle_{B}$ is large, integrating only the expression for the small- $\theta$ part $\langle D\rangle_{B}^{S}$ over the domain situated close to the loss cone angle $\alpha_{L C}$ and the large- $\theta$ part $\langle D\rangle_{B}^{l}$ between about $\pi / 12$ and $\pi / 2$. For the small- $\theta$ part, $T\left(\alpha_{0}\right)$ can be approximated by $T\left(\alpha_{L C}\right) \sim 1.3$, so that $1 /\left(\langle D\rangle_{B}^{S} \tan \alpha_{0}\right) \propto 1 / \tan \alpha_{0}$ can be integrated analytically from the loss cone angle $\alpha_{L C}$ up to an angle $\alpha_{+}$comprised between $\alpha_{L C}$ and $\pi / 2$. One can take $\alpha_{+} \sim 7 \alpha_{L C}$, as numerical calculations show that the integral does not increase significantly at larger $\alpha_{0}$ for $L=4$ to 6.5 . Finally, one obtains respectively for the small- $\theta$ part alone and for the total $g(\theta)$ distribution:

$$
\begin{gathered}
\tau_{s} \approx \sigma \frac{\ln \left(\frac{\sin \alpha_{+}}{\sin \alpha_{L C}}\right)}{2\langle D\rangle_{B}^{s}}, g=g_{s} \\
\tau_{\text {total }} \approx \tau_{\text {landau }}+\sigma \frac{\frac{1}{2} \ln \left(\frac{\sin \alpha_{+}}{\sin \alpha_{L C}}\right)}{\left\langle D^{+}\right\rangle_{B}^{l}\left(\frac{\pi}{4}\right)+\langle D\rangle_{B}^{s}}, g=g_{s}+g_{l}
\end{gathered}
$$

where the Landau part (minimum of $D$ ) above $\alpha_{0}^{\operatorname{Max}}(n=-1)$ is given by Mourenas and Ripoll [2012] with $\left\langle D_{0}\right\rangle_{B} / 2$ and an additional factor for large $\omega_{m} / \Omega_{c 0}$ :

$$
\begin{aligned}
\tau_{\text {landau }} \approx & \frac{B_{0}^{2}\left|1-\omega_{m} / \Omega_{c 0}\right|^{-3 / 2}}{2.4 \gamma B_{\text {wave }}^{2} G_{\omega 0}\left(\omega_{m}\right) \Delta \omega}\left(\frac{p \Omega_{p e}}{\Omega_{c 0}}\right)^{4} \frac{\sigma_{\theta}}{\sin ^{3} \alpha_{M 0}} \\
& \times \frac{\frac{\pi}{2}-\alpha_{0}^{\operatorname{Max}}-\frac{2}{3} \sin \left(2 \alpha_{0}^{\operatorname{Max}}\right)-\frac{1}{12} \sin \left(4 \alpha_{0}^{\operatorname{Max}}\right)}{g_{s}\left(\theta_{M 0}\right)+\min \left(\frac{\left(\sigma_{\theta} p \varepsilon_{m 0}\right)^{3}}{11}, \frac{1}{p \varepsilon_{m 0} \sigma_{\theta}}\right)}
\end{aligned}
$$

with $\sigma_{\theta}=\tan \Delta \theta \sim 1$ in the small- $\theta$ part, where

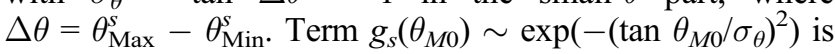
assumed for the Landau part, where $\tan \theta_{M 0} \sim 1.84 / p \varepsilon_{m 0}$ and $\alpha_{M 0}$ is given in section 2.5. A theoretical estimate of the threshold for increased lifetimes due to a sufficiently deep minimum of $\langle D\rangle$ between the peaks of first cyclotron and 


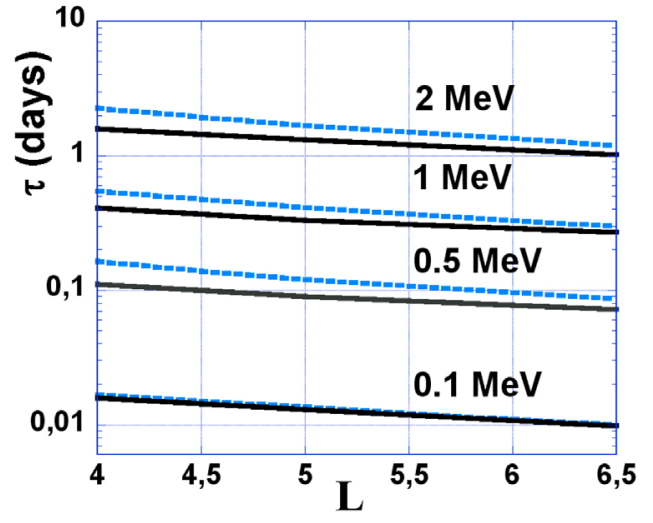

Figure 4. Lifetimes of $0.1 \mathrm{MeV}, 0.5 \mathrm{MeV}, 1 \mathrm{MeV}$, and $2 \mathrm{MeV}$ (from bottom to top) electrons interacting with small- $\theta$ day-side low-frequency chorus waves in the outer zone of the radiation belts, as a function of $\mathrm{L}$. The numerical parameterization of equation (3) of Shprits et al. [2007] is shown (black solid line) as well as analytical estimate (10) and (16) (blue dashed line).

Landau resonances can be obtained from equations (16) and (17). It reads as

$$
p<\frac{0.65}{\sigma_{\theta}^{2 / 5}}\left(\frac{\Omega_{c 0}^{5 / 2}}{\Omega_{p e} \omega_{U}^{3 / 2}}\right)\left(1-\frac{\omega_{U}}{\Omega_{c 0}}\right)
$$

giving $E<100 \mathrm{keV}$ in the case where $\omega_{m} / \Omega_{c 0}=0.2$ and $\Omega_{p e} / \Omega_{c 0}=4.5$, in good agreement with the full numerical solution (see Figure 7). This minimum of $\langle D\rangle$ is deeper when the pitch angle interval between the maxima of the Landau and first Cyclotron resonances is wider, for larger values of $\cos \alpha_{M, 0}-\cos \alpha_{0}^{\operatorname{Max}}(n=-1)$. It corresponds to small energy, frequency and density, and large magnetic field, as already apparent in numerical results shown in Figure 10 of Lyons et al. [1972].

[29] Considering only small- $\theta$ diffusion from equation (10) at $L \geq 4$, with electron density $N_{e} \propto L^{-4}$ [as in Sheeley et al., 2001], and with a ratio $\omega_{m} / \Omega_{c 0}$ kept fixed, we find that in the relevant regime of interaction with small- $\theta$-only chorus waves, electron lifetimes scale roughly as:

$$
\tau \propto \frac{p^{3 / 2} \gamma \omega_{m}^{7 / 9} \Omega_{p e}^{14 / 9} \ln \left(\sin \alpha_{L C}\right)}{\Omega_{c 0}^{12 / 9}} \propto \frac{E^{2}}{L}
$$

for

$$
\begin{aligned}
& p \varepsilon_{m 0}>1.84 / \sigma_{\theta} \\
& \gamma p^{4 / 3}>\frac{\Omega_{c 0}^{17 / 6} \sqrt{\sigma_{\theta}}}{12 \Omega_{p e}^{4 / 3} \omega_{m}^{3 / 2}} .
\end{aligned}
$$

[30] With the ratio $\omega_{m} / \Omega_{c 0}$ fixed, lifetimes should vary roughly like $E^{2} / L$ at high energies and/or $L$ (as actually observed in Figure 1 of Shprits et al. [2007]) for $1 \mathrm{MeV}$ electrons scattered by chorus waves such that $\theta \sim 0$.

[31] This behavior stems from the integrand in (9) being roughly $\left(\Omega_{c 0} / \Omega_{c}\left(\lambda_{R}\right)\right)^{2} \sim 1 /\left(p \varepsilon_{m 0}\right)^{3 / 2}$ from resonance condition (A1), corresponding to decreasing $\langle D\rangle$ at higher resonant latitudes, i.e. at large energy, frequency and density, and at smaller magnetic field values, in agreement with numerical results in Figure 10 of Lyons et al. [1972]. Note also that lifetimes $\tau$ in equation (19) do not depend upon $\Delta \omega$ and $\Delta \theta$ at moderate and large $E$, only increasing with $\Delta \omega$ at very low energies.

[32] An insensitivity of $\left\langle D_{-1}\right\rangle_{B}^{s}$ at $\alpha_{0}<50^{\circ}$ to the chorus wave-normal distribution has already been emphasized from recent numerical simulations [Shprits and $N i, 2009$ ] performed in the special case of small- $\theta$ diffusion $\left(<45^{\circ}\right)$. It leads directly to a similar insensitivity of $\tau$. However, properly taking into account large- $\theta$ diffusion terms (12) based on CLUSTER chorus wave observations [Agapitov et al., 2011] leads to more complicated dependencies upon the parameters at low energy. Nevertheless, at high energy where $\tau_{\text {total }} \ll \tau_{s}$, one gets

$$
\tau_{\text {total }} \propto \gamma p \Omega_{p e} \propto E^{3 / 2} / L^{2} .
$$

[33] Comparing (19) and (20), one can see that $\tau_{\text {total }} / \tau_{s} \propto E^{-1 / 2} L^{-1}$ so that the reduction of lifetimes is more important at large $L$ and high energy. A striking new feature is that the lifetimes are strongly reduced in realistic cases, by as much as a factor of 10 at high energy. It is mainly due to the fact that the number of large- $\theta$ resonances (7) increases with energy and density.

[34] Momentum and energy diffusion coefficients can easily be obtained from the above $D$ pitch angle diffusion coefficients [see Lyons, 1974; Glauert and Horne, 2005]. A significant decrease of acceleration timescales is expected when large- $\theta$ diffusion by lower-band chorus becomes important at $E>30 \mathrm{keV}$ on the day-side in the outer belt. This will be the topic of a future work.

\section{Comparisons With Full Numerical Simulations and Discussion}

[35] First, the analytical estimate (16) of electron lifetimes for small- $\theta$ diffusion only, calculated with diffusion coefficient's expression (10), is compared with the numerical calculations of Shprits et al. [2007] for interaction of electrons with the low-frequency band of chorus whistler waves dominant on the day-side in the outer radiation belt, where $\omega_{m} / \Omega_{c 0} \sim 0.2$. We use the same initial conditions: $100 \mathrm{pT}$ storm-time chorus waves and dayside plasma trough density $N_{e} \sim 100(3 / L)^{4} \mathrm{~cm}^{-3}$ for $L \geq 3$ from Sheeley et al. [2001]. In order to compare with the small- $\theta$ PPA approximation used in Shprits et al. [2007], we take here $\theta_{\mathrm{Max}}<\pi / 4$. Comparisons with equation (3) of Shprits et al. [2007] are restricted to $E \geq 0.1 \mathrm{MeV}$ because their parameterization is stated to remain accurate within about $20 \%$ for $0.1 \mathrm{MeV} \leq E \leq 2 \mathrm{MeV}$ only. Note that equation (3) of Shprits et al., 2007 reads $\tau=0.039 E^{2}+0.047 / L+1.41 E^{2} / L$, which is consistent with their Figures 1 and 2 . Moreover, chorus waves are assumed to be present up to $\lambda \approx 35^{\circ}$, peaking between $\lambda \approx 15^{\circ}$ and $\lambda \approx 30^{\circ}$.

[36] The numerical solutions are fairly recovered by the analytical estimate (10) and (16) in Figure 4. In particular, one finds that lifetimes vary roughly as $\sim E^{2} / L$, especially for $L>4.5$. The discrepancy is most often much smaller than $30 \%$, which is much smaller than the inaccuracies associated to both the electron density profile and the wave amplitude models. Figures 5 and 6 display the variation of analytical 


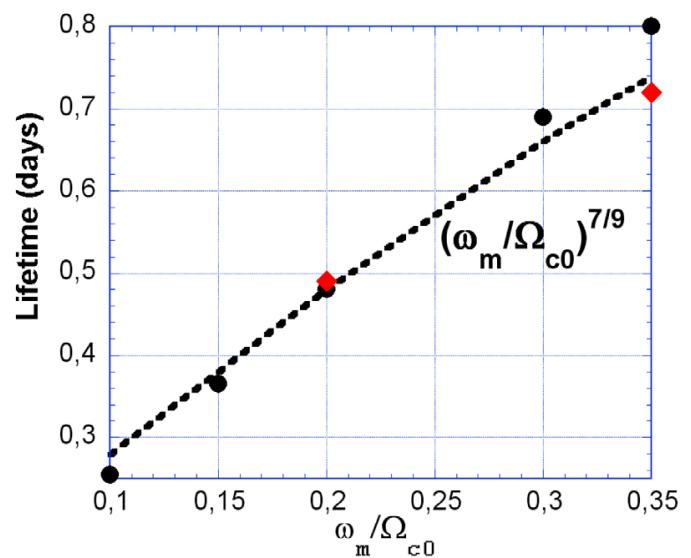

Figure 5. Lifetime variation as a function of the central chorus frequency $\omega_{m}$ at $L=4.5$ for $E=1 \mathrm{MeV}$, with the same other parameters as in Figure 4. The analytical estimate (10) and (16) is plotted (black circles) as well as the scaling $\omega_{m}^{7 / 9}$ from equation (19) (dashed line) and the full numerical results (red diamonds).

and numerical lifetimes for $\theta<\pi / 4$ as a function of density and frequency. The rough scaling (19) deduced from (16) appears approximately correct, with analytical lifetimes varying like $\tau \propto\left(N_{e} \omega_{m}\right)^{7 / 9}$ over a large parameter range. These variations were not provided by Shprits et al. [2007] in their parameterization based on a large number of numerical simulations.

[37] We also compare lifetime estimates with or without the large- $\theta$ part (16) with our full numerical calculations based on the CLUSTER measurements (description of numerical code can be found in Appendix E). Pitch angle diffusion coefficients are calculated with $g(\theta)$ consisting of $g_{l}(\theta)$ and $g_{s}(\theta)$ parts. The comparisons are performed for two different values of the ratio $\Omega_{p e} / \Omega_{c 0}$ corresponding to different $L$ values above $L=4$ (or different natural conditions) and also for two different values of the mean chorus frequency $\omega_{m} / \Omega_{c 0}=0.2$ and $\omega_{m} / \Omega_{c 0}=0.35$, with the same other parameters as before (see Figure 7).

[38] One can see that lifetime estimate (16) for the small- $\theta$ part is in fair agreement with the full numerical results for both $\omega_{m}$ and $\Omega_{p e}$ values. The $\tau_{\text {total }}$ estimate for the complete small- $\theta$ and large- $\theta$ distribution is also in relatively good agreement with the full numerical results. The discrepancy between analytical and numerical lifetimes is often much smaller than a factor of 2 , especially at high energy $E>400 \mathrm{keV}$. Considering the simplifications done in the course of the analytical calculations and the rough average over the small and large $\theta$-parts made in (14), it is a satisfactory result. The discrepancy between the analytical estimates and the full numerical results is actually much smaller than the uncertainties stemming from outer-belt density modeling and intrinsic variability [e.g., see Sheeley et al., 2001; Denton et al., 2002] as well as from chorus waves intensity models [Meredith et al., 2001; Horne et al., 2005; Shprits et al., 2007; Li et al., 2011] which show a large spread of measured intensities in relation to the highly variable geomagnetic activity. Since the analytical lifetimes lie well within the uncertainty range surrounding the full numerical results, they could prove useful (albeit approximate) substitutes inside global radiation belt codes relying on similar density and wave intensity models for more quickly evaluating energetic electron fluxes (at least in the parameter range studied here). On the other hand, full numerical solutions should obviously be preferred for any simulation aiming at accurately reproducing specific storm-related events when the different plasma/wave parameters can be determined more precisely from satellite measurements.

[39] Lifetimes in Figure 7 are one order of magnitude smaller with large- $\theta$ waves included (red crosses and solid blue line) than with small- $\theta$ waves only (diamonds and dashed blue line). At large energy $E>400 \mathrm{keV}$, we observe a significant input of higher-order cyclotron resonances, as expected from equation (7). This strong reduction of timescales suggests that the storm-time mechanism of energy transfer from low-energy injected electrons to high-energy ones through chorus excitation by loss cone instability [e.g., see Horne et al., 2005] could become even more efficient at moderate to high latitudes in the presence of highly oblique waves. This is left as the topic of a further work. Comparing lifetimes $\tau_{\text {total }}$ of electrons to their azimuthal drift period $\tau_{\text {drift }}$ [Hamlin et al., 1961], one finds that $\tau_{\text {total }} / \tau_{\text {drift }}<1 / 4$ at $E \sim 15 \mathrm{keV}$ to $200 \mathrm{keV}$ for $100 \mathrm{pT}$ dayside storm-time chorus waves such that $\omega_{m} / \Omega_{c 0} \sim 0.35$ (below $15 \mathrm{keV}$, the Landau term (17) leads to a rapidly increasing $\left.\tau_{\text {total }}\right)$. Such electrons should generally be lost during their first drift from dawn to noon. The actual large- $\theta$ lifetimes of low-energy electrons are therefore such as plotted in Figure 7 and given by (16), while large- $\theta$ lifetimes of higher-energy electrons and small- $\theta$-only lifetimes must be multiplied by a factor $\sim 4$ to account for the $25 \%$ occurrence rate of the waves over a full drift period [Shprits et al., 2007]. Let us consider moderate-latitude chorus of about $140 \mathrm{pT}$ at $L=4.5$ and $100 \mathrm{pT}$ at $L=6.5$. The corresponding large- $\theta$ lifetimes $\tau_{\text {total }}(\mathrm{s}) \sim\left(\gamma p / L^{2}\right)\left(10^{4} / B_{w}[\mathrm{pT}]\right)^{2}$ are 1 minute at $15 \mathrm{keV}$ and 3 min at $100 \mathrm{keV}$. Numerical results in Figure 7 imply nearly 3 times smaller lifetimes at $E<100 \mathrm{keV}$. It is worth noting that the resulting minimum timescales of $20 \mathrm{~s}$ to $60 \mathrm{~s}$ for $15 \mathrm{keV}$ to $100 \mathrm{keV}$ electrons are similar to the observed

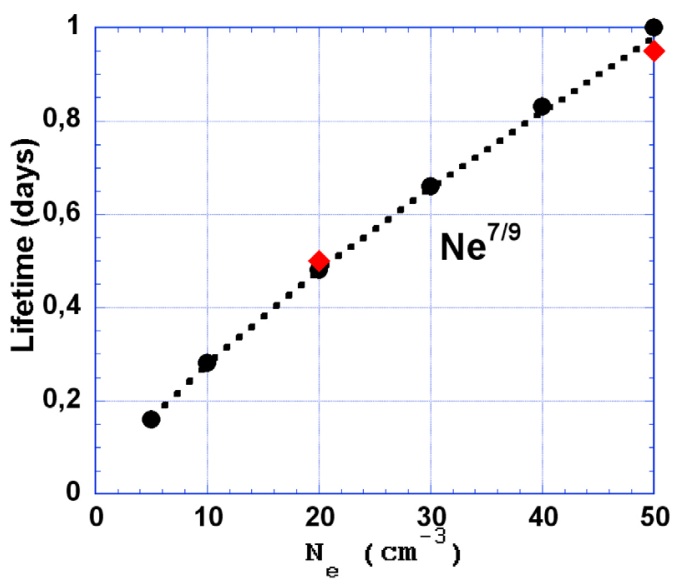

Figure 6. Lifetime variation given by (10) and (16) as a function of the electron density $N_{e}$ at $L=4.5$ for $E=1 \mathrm{MeV}$, with the same other parameters as in Figure 4, and the scaling $N_{e}^{7 / 9}$ from equation (19) (dashed line) and the full numerical results (red diamonds). 

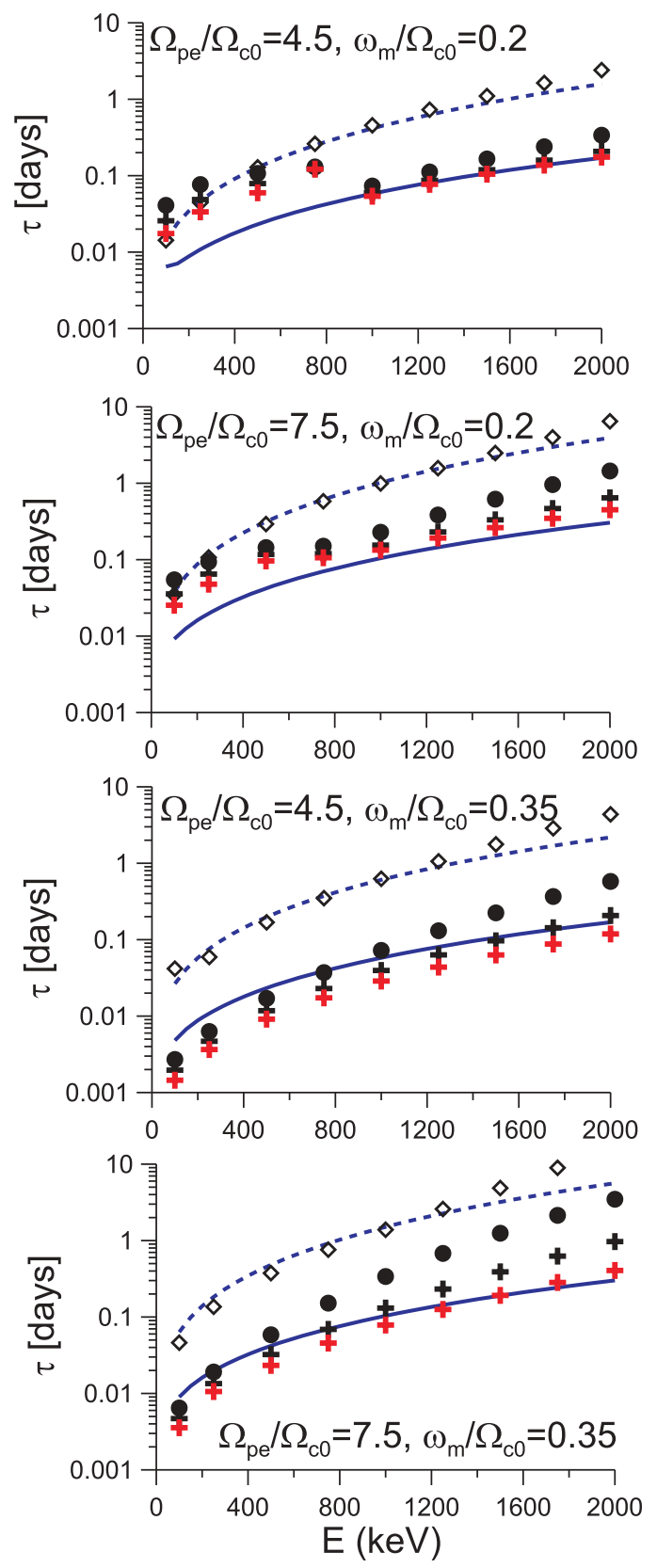

Figure 7. Lifetime variations given by (16) with large- $\theta$ part (solid blue curve), without large- $\theta$ part (dashed blue curve) are compared with those obtained by numerical scheme (filled circles show results obtained with large- $\theta$ part and $|n| \leq 5$, black crosses correspond to $|n| \leq 10$, red crosses correspond to $|n| \leq 20$, and diamonds show results obtained without large- $\theta$ for $|n| \leq 5$; $\tau$ is calculated for $\alpha_{+}=\pi / 2$ ).

periods of pulsating auroras, which were recently shown to be highly correlated with lower-band chorus [Nishimura et al., 2011]. These timescales are also similar to, or lower than strong diffusion timescales of isotropization $\tau_{s d} \sim 1.8 L R_{E} \gamma /$ ( $p c \alpha_{L C}^{2}$ ), where $R_{E}$ is the Earth's radius [Lyons, 1973; Schulz, 1974]. Quasi-linear scattering of substorm-injected electrons in the loss cone by very oblique chorus waves, leading successively to enhanced wave growth [Horne et al., 2005], loss cone filling, and instability quenching on similar timescales
[Davidson, 1986], might therefore account for the pulsation periods of some dawnside auroras, lending further credibility to models of chorus-generated pulsating aurora [see Nishimura et al., 2011, and references therein]. On a more global scale, the dayside large- $\theta$ diffusion rates plotted in Figure 8 are much stronger at small pitch angles $\alpha_{0}<\pi / 4$ than at larger values. As a result, the outer belt eastward-drifting energetic electron population could exhibit a predominantly perpendicular anisotropy on the dayside at times of low disturbances, while showing some isotropization in the strong diffusion regime at low energy during very active periods. Similar anisotropy variations have actually been observed, although drift shell-splitting is also believed to produce a perpendicular dayside anisotropy at large $L$ [see $G u$ et al., 2011; Borovsky and Denton, 2011, and references therein].

[40] In Figure 8, bounce-averaged diffusion coefficients obtained with the full wave-normal angle distribution and with the small- $\theta$ part only, are displayed for different energies, for the same parameters as before $(L \sim 4.5$ and $100 \mathrm{pT}$ chorus). It is worth pointing out in Figure 8 the deep minimum in total diffusion rate between the Landau and first cyclotron resonances at $\alpha_{0} \sim 75^{\circ}$ for small mean frequency $\omega_{m} / \Omega_{c 0}=0.2$ and at low energy $E<200 \mathrm{keV}$. Such a deep minimum increases lifetimes [Albert and Shprits, 2009]. At $E=100 \mathrm{keV}$, this minimum being slightly wider when large$\theta$ waves are included, it leads to a total lifetime (including small and large $\theta$-parts and plotted in Figure 7 as red crosses) climbing slightly above the small- $\theta$ lifetime (diamonds symbols in Figure 7), contrary to what happens in all the other examples shown. This is in good agreement with equation (18), which gives a threshold $E \sim 100 \mathrm{keV}$ for this effect, although the shape and values of the lifetimes are not precisely recovered by equations (16) and (17).

[41] For $\omega_{m} / \Omega_{c 0}=0.35$, the actual minimum of $\langle D\rangle \tan \alpha_{0}$ in the numerical simulations does occur at $\alpha_{0} \geq 20^{\circ}$ as assumed in the analytical developments. For $\omega_{m} / \Omega_{c 0}=0.2$, however, improperly neglecting high-order resonances $|n|>5$ leads to a clear minimum at smaller pitch angles, which increases significantly the numerical lifetimes (for instance at $100-300 \mathrm{keV}$ in Figure 7). These high- $n$ resonances occur at high latitudes for $\theta \sim \theta_{r}$ near the loss cone. High-order resonances must therefore always be taken into account, even at low energy.

[42] To assess the impact of high- $n$ resonances we plot diffusion coefficients obtained numerically with $|n| \leq 5$, $|n| \leq 10$ and $|n| \leq 20$ in Figure 9. High- $n$ resonances are involved into diffusion only for particle with small to moderate pitch angles $\alpha_{0}<60^{\circ}$. These particles can reach highlatitudes where mean value of $g(\theta)$ distribution is situated relatively close to $\theta_{r}$, i.e. $\theta_{m}+\Delta \theta^{l}>\theta_{r}$, where $\Delta \theta^{l} \approx \theta_{\text {Max }}^{l}-\theta_{\text {Min. }}^{l}$. In this case the number of resonances increases rapidly as seen from equation (7). Note also that the analytical estimate $\langle D\rangle$ agrees well with the numerical solution for $\alpha_{0}>15^{\circ}$ in Figure 9. The ratio $\Pi$ of diffusion coefficients calculated for $|n| \leq 20$ and $|n| \leq 5$ is also plotted in Figure 9, as well as our corresponding analytical estimate $\sim 2 N_{r} \cos \alpha_{0}\left(\theta_{g 0}, \omega_{m}\right) / 5$ (see Appendix D). It demonstrates that the related increase of diffusion is more important at $\alpha_{0}<45^{\circ}$, as expected from equation (7). This increase of diffusion is rather well reproduced by the analytical estimate. From equation (7), one also finds 

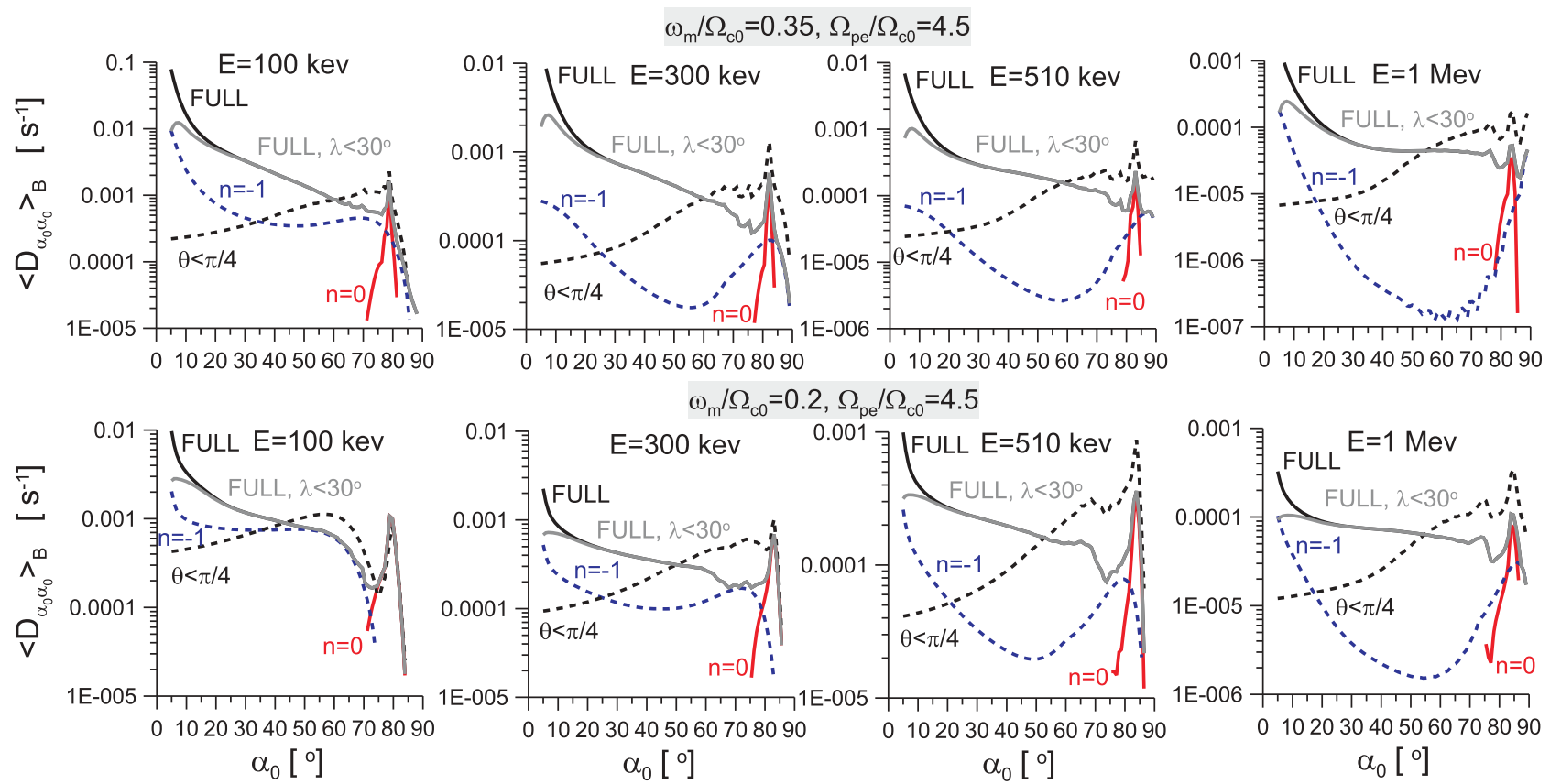

Figure 8. Numerical bounce-averaged diffusion coefficients as a function of equatorial pitch angle for $|n| \leq 5$. The full solution with $g(\theta)=g_{s}(\theta)+g_{l}(\theta)$ is plotted (solid line) as well as the full solution with latitudes bounded at $30^{\circ}$ (grey line), and the $n=0$ and $n=-1$ resonances alone (solid red and dashed blue lines). The solution with $g=g_{s}(\theta)$ is also shown for $\theta<\pi / 4$ (dashed black line).

$N_{r} \leq 10$ for $\alpha_{0} \sim 45^{\circ}$ in our test-cases at $E<2 \mathrm{MeV}$, except for $\omega_{m} / \Omega_{c 0}=0.35$ and $\Omega_{p e} / \Omega_{c 0}=7.5$ where $N_{r} \leq 14$.

\section{Conclusions}

[43] In this paper, analytical estimates of energetic electron lifetimes in the radiation belts have been derived, based on the use of the Lyons et al. [1972] quasi-linear model with the weighted-average reformulation of Albert [2007], on the approximation of the Bessel functions by their envelop maximum, and on the assumption of a resonance maximum occurring at the mean frequency $\omega_{m}$ (for $\left.\Delta \omega \leq \omega_{m} / 2\right)$. We consider low-frequency $\left(\Omega_{c i} \ll \omega<\Omega_{c} / 2, \omega \Omega_{c} \ll \Omega_{p e}^{2}\right)$ oblique chorus whistler waves, such as observed in the outer belt by CLUSTER [Agapitov et al., 2011]. The analytical model captures quite well many features of the full-numerical solution, and provides new lifetime scaling laws as a function of energy, $L$-shell, density and wave frequency, bringing a better understanding of the simulations results. In particular, numerical as well as analytical calculations presented here demonstrate that timescales can be strongly reduced when the waves reach large wave-normal angles, between the Gendrin and resonance angles. Such very oblique chorus waves are often present at low to moderate latitudes, where they represent from $10 \%$ to $50 \%$ of the occurrences [Hayakawa et al., 1990; Santolik et al., 2009; Verkhoglyadova and Tsurutani, 2008; Agapitov et al., 2011; Haque et al., 2010, 2011]. Chorus waves can be directly generated with high wave-normal angles inside the source region [Santolik et al., 2009; Haque et al., 2011] or experience an increase of their wave-normal angle as they propagate along a geomagnetic field line away from the source region, believed to be located close to the equator [Lauben et al., 2002; Chum and Santolik, 2005; Bortnik et al., 2011]. Scattering of moderate pitch angle electrons into the loss cone appears to be much more efficient for such very oblique chorus waves, potentially reaching the strong diffusion limit, which might explain some features of pulsating

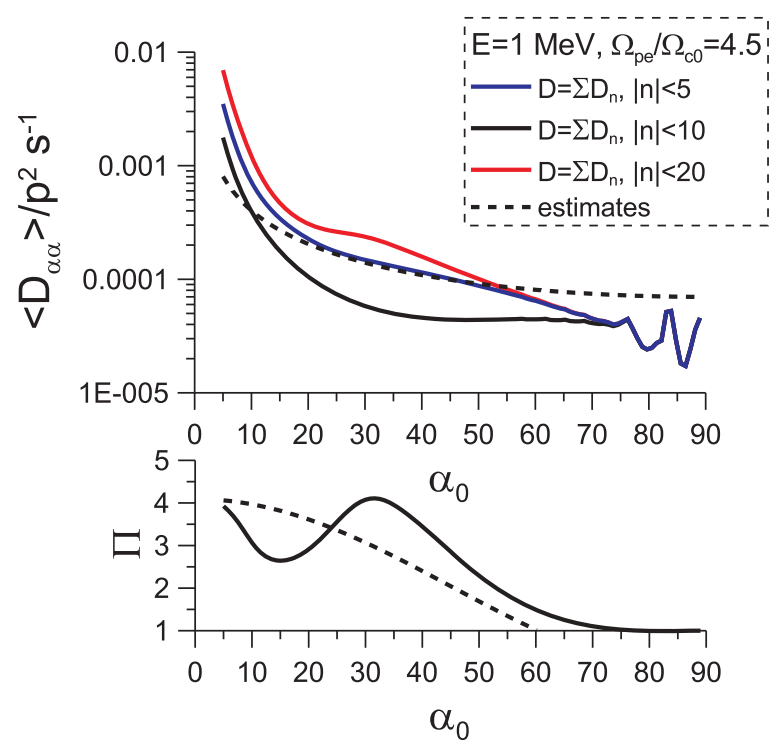

Figure 9. (top) Numerical bounce-averaged diffusion coefficients for $\theta$ distribution $g(\theta)=g_{s}(\theta)+g_{l}(\theta)$ and (bottom) ratio of $|n| \leq 20$ over $|n| \leq 5$ diffusion coefficients as a function of equatorial pitch angle for $1 \mathrm{MeV}$ electrons. The analytical estimates of $\langle D\rangle$ and $2 N_{r} \cos \alpha_{0} / 5$ are also plotted (dashed black lines). 
auroras as well as translate into a predominantly perpendicular anisotropy of relativistic outer belt electrons on the dayside. Further work will be needed to examine in details all the consequences of this increased scattering, concerning in particular the energization of relativistic electrons [Horne et al., 2005; Li et al., 2007; Summers et al., 2007b].

[44] Energy diffusion, radial transport, and finite burst durations must also be taken into account in the highly variable environment of the outer belt, requiring the use of global multidimensional codes [Shprits et al., 2008; Varotsou et al., 2008; Fok et al., 2011]. In this growing field of space weather forecasting for satellite warning, the proposed analytical estimates might prove useful for quickly evaluating the sensitivity of particle fluxes calculated by global radiation belt codes to often insufficiently known wave and plasma parameters. Nevertheless, one should bear in mind that the proposed model has only been partially validated against numerical simulations in a restricted parameter range. Analytical estimates cannot be expected to replace full numerical calculations, which are becoming more and more rapid with the advent of massively parallel supercomputers and will always remain the gold standard of radiation belts physics.

[45] Further tests of the accuracy of our analytical estimates are planned in the future. Still, magnetosonic, EMIC and high-frequency chorus waves are also important above $L \sim 4$ [Summers et al., 2007a, 2007b; Li et al., 2007] and have not been treated here. The full numerical resolution of the Fokker-Planck diffusion equation is required in the highly variable environment of the outer radiation belt to take into account pitch angle diffusion by all these waves as well as energy diffusion, radial transport and finite burst durations.

\section{Appendix A: Derivation of $\Delta_{n} / \Gamma$}

[46] Here we derive an expression for the resonant value of pitch angle $\alpha_{R}$. Combining the simplified dispersion relation with the resonance condition leads to the relation

$$
\cos \alpha_{R}=\frac{|n|\left|1+\frac{\gamma \omega_{m}}{n \Omega_{c}}\right|\left|1-\frac{\omega_{m}}{\Omega_{c} \cos \theta}\right|^{1 / 2}}{p \varepsilon_{m} \sqrt{\cos \theta}}
$$

with $\varepsilon_{m}(\lambda)=\left(\Omega_{p e} / \Omega_{c}\right) \sqrt{\omega_{m} / \Omega_{c}}$, where $\lambda$ is the geomagnetic latitude, $p=v \gamma /\left(m_{e} c\right)$ is the electron momentum, $m_{e}$ the electron mass, $c$ the speed of light. Considering adiabatic motion along the bounce trajectory, one can rewrite the above equation as

$$
\frac{\cos \alpha_{R}}{\sin ^{3} \alpha_{R}}=\frac{|n|\left|1+\frac{\gamma \omega_{m}}{n \Omega_{c}}\right|\left|1-\frac{\omega_{m}}{\Omega_{c} \cos \theta}\right|^{1 / 2}}{p \varepsilon_{m 0} \sqrt{\cos \theta} \sin ^{3} \alpha_{0}}
$$

where $\varepsilon_{m 0}$ is the value of $\varepsilon_{m}$ at the equator and $\alpha_{0}$ is the equatorial pitch angle (we use the relation $\sin ^{2} \alpha=\sin ^{2} \alpha_{0}\left(\Omega_{c} / \Omega_{c 0}\right)$ where $\Omega_{c 0}$ is the equatorial value of $\Omega_{c}$ ). We assume in equation (A1) that the density remains roughly constant along a geomagnetic field line up to a maximum latitude of interaction of about $35^{\circ}$ [Denton et al., 2002; Horne et al., 2005]. We use subscript " 0 " for equatorial values of variables.

[47] In a previous paper [Mourenas and Ripoll, 2012], we simplified the different relations and the diffusion coefficients expressions by taking the limits $\gamma \omega_{m} / \Omega_{c} \ll 1$ and $\cos \theta \sim 1$. While these are reasonable and convenient approximations for moderately oblique plasmaspheric hiss waves such that $\omega_{m} / \Omega_{c 0}<0.06$ and $E<3 \mathrm{MeV}$, it is not anymore always correct for outer zone chorus waves interacting with energetic particles, where higher-order terms must be kept.

[48] Keeping terms $\sim \gamma \omega_{m} / \Omega_{c}$, we can rewrite the term at the denominator of equation (2) as

$$
\left|1-\frac{\left(\partial \omega / \partial k_{\|}\right)_{r}}{v \cos \alpha}\right| \approx\left|1-2 \frac{1-\frac{\omega_{m}}{\Omega_{c} \cos \theta}}{1+\frac{n \Omega_{c}}{\gamma \omega_{m}}}\right|
$$

where $\left(\partial \omega / \partial k_{\|}\right)_{r}$ is the parallel component of the group velocity at resonance. We have also $\Gamma=\frac{1}{2}|\mu|^{3}\left|\Omega_{c} \cos \theta /\left(\Omega_{c} \cos \theta-\omega_{m}\right)\right|$. Using the resonance condition, it can be rewritten

$$
\Gamma=\frac{\left|1+\frac{n \Omega_{c}}{\gamma \omega_{m}}\right|^{3}}{2\left|1-\frac{\omega_{m}}{\Omega_{c} \cos \theta}\right|\left|v_{\|} \cos \theta / c\right|^{3}}
$$

and it can be further simplified at small $\theta$. Simplified expressions of $\Phi_{n}^{2}$ valid at small and large values of $\theta$ can be derived from the exact formula given in equations (9), (11), and (12) of Lyons [1974]. To this aim, let us first recall that $\theta$ is necessarily smaller than the (maximum) resonance angle $\theta_{r}=\arccos \left(\omega_{m} / \Omega_{c}\right)$, so that $\Omega_{c} \cos \theta / \omega_{m}>1$. Assuming further that $\left(\omega_{m} / \Omega_{c}\right)^{2} \ll 1$ and $\Omega_{c} / \omega_{m} \gg \cos \theta$ at large $\theta$, such that $\theta \geq \theta_{g}$, with $\theta_{g}=\arccos \left(2 \omega_{m} / \Omega_{c}\right)$ the Gendrin angle at which the waves group velocity in the magnetic field direction reaches its maximum, the waves being guided along the field line [Gendrin, 1961]. It yields the desired expressions

$$
\Phi_{n}^{2} \approx\left\{\begin{array}{l}
\frac{\left(\left(1+\cos \theta-\frac{\omega_{m}}{\Omega_{c}}\right) \mathrm{J}_{n+1}+\left(1-\cos \theta+\frac{\omega_{m}}{\Omega_{c}}\right) \mathrm{J}_{n-1}\right)^{2}}{8 \cos ^{2} \theta\left(1-\frac{\omega_{m}}{\Omega_{c} \cos \theta}\right)^{2}} \\
\theta \geq \max \left(\theta_{g}, \frac{1}{4} \pi\right) \\
\frac{\left((1+\cos \theta) \mathrm{J}_{n+1}+(1-\cos \theta) \mathrm{J}_{n-1}\right)^{2}}{8 \cos ^{2} \theta} \\
\theta<\max \left(\theta_{g}, \frac{1}{4} \pi\right)
\end{array}\right.
$$

[49] The ratio $\Delta_{n} / \Gamma$ is readily obtained from equation (A2) and equation (2) in the limit $\sin ^{2} \alpha<|n| \Omega_{c} /\left(\gamma \omega_{m}\right)$ :

$$
\frac{\Delta_{n}}{\Gamma} \approx\left\{\begin{array}{l}
\frac{\pi\left(\left(1+\cos \theta-\frac{\omega_{m}}{\Omega_{c}}\right) \mathrm{J}_{n+1}+\left(1-\cos \theta+\frac{\omega_{m}}{\Omega_{c}}\right) \mathbf{J}_{n-1}\right)^{2}}{8\left|1-\frac{\omega_{m}}{\Omega_{c} \cos \theta}\right| 1-\frac{2 \omega_{m}}{\Omega_{c} \cos \theta}-\frac{n \Omega_{c}}{\gamma \omega_{m}} \mid\left(1+\frac{\gamma \omega_{m}}{n \Omega_{c}}\right)^{2}}, \\
\theta \geq \max \left(\theta_{g}, \frac{1}{4} \pi\right) \\
\frac{\pi\left((1+\cos \theta) \mathrm{J}_{n+1}+(1-\cos \theta) \mathrm{J}_{n-1}\right)^{2}\left|1-\frac{\omega_{m}}{\Omega_{c} \cos \theta}\right|}{\theta\left|1-\frac{2 \omega_{m}}{\Omega_{c} \cos \theta}-\frac{n \Omega_{c}}{\gamma \omega_{m}}\right|\left(1+\frac{\gamma \omega_{m}}{n \Omega_{c}}\right)^{2}}, \\
\theta<\max \left(\theta_{g}, \frac{1}{4} \pi\right)
\end{array}\right.
$$


[50] We recover $\Delta_{n} / \Gamma \sim(\pi / 2) \mathrm{J}_{0}^{2} \gamma \omega_{m} / \Omega_{c}$ for $n=-1$ in the limits $\Omega_{c} /\left(\gamma \omega_{m}\right) \gg 1$ and $\theta \sim 0$, as in Mourenas and Ripoll [2012]. From equation (A3), it is easy to see that the most important contributions to diffusion come from the maxima of the Bessel functions. Examining the denominator of equation (A3), one can see that three different singularities may occur, which will be investigated in this paper. It is already clear from equation (A3), however, that in the large$\theta$ range, $\Delta_{n} / \Gamma$ can experience a large increase near the resonance angle as compared to the small- $\theta$ case.

\section{Appendix B: Resonance Domains}

[51] It has already been shown in Mourenas and Ripoll [2012] that the resonant wave-normal angle is linked to the harmonic number and the pitch angle at the Bessel function's maximum, where the contribution to $D_{n}^{\alpha \alpha}$ is dominant at moderate latitudes. Indeed, the first maximum of the Bessel functions occurs at $x=x_{M}(n) \approx|n|+0.81|n|^{1 / 3} \approx|n|+1$, except when $n=0$ [Abramowitz and Stegun, 1972]. The error in the $x_{M}(n) \approx n+1$ approximation is less than $1 / n$ at large $n$, which is small. Taking $\Phi_{n}^{2} \propto \mathrm{J}_{|n|-1}^{2}$ and resonance relation one obtains $\tan \alpha \tan \theta \sim 1$ at the Bessel maxima, except for $\mathrm{J}_{0}$, in which case $\tan \alpha \tan \theta<1$. This gives

$$
\left\{\begin{array}{l}
\alpha_{R, M} \approx \frac{1}{2} \pi-\theta_{R},|n|>1 \\
\alpha_{R, M} \leq \frac{1}{2} \pi-\theta_{R},|n|=1
\end{array}\right.
$$

[52] Neglecting the phase terms, classic asymptotic expansions of Bessel functions for large arguments $x>n$ and ascending series at small $x \ll n$ can be used. Since the first maximum of the $\mathrm{J}_{n}(x)$ functions occurs at $x=x_{M}(n) \sim|n|+1$ (except when $n=0$ ), the Bessel functions can be rewritten as

$$
\begin{aligned}
& \mathrm{J}_{|n|-1}^{2} \approx \frac{2}{\pi|n|} \frac{x_{M}}{x}, x>x_{M}(|n|-1) \\
& \mathrm{J}_{|n|-1}^{2} \approx \frac{2}{\pi|n|}\left(\frac{x}{x_{M}}\right)^{2|n|-2}, x<x_{M}(|n|-1),|n| \neq 1
\end{aligned}
$$

[53] Also we have $\mathrm{J}_{0}^{2}=1$.

[54] Let us first consider the domain $\theta<\min \left(\theta_{\mathrm{Max}}^{s}, \theta_{g}, \pi / 4\right)$. For $\sqrt{\cos \theta} \sim 1$ and at small equatorial pitch angles close to loss cone value $\alpha_{0} \sim \alpha_{L C}$ such that $\sin \alpha_{0}\left(p \varepsilon_{m 0} / \mathrm{l} n+\gamma \omega_{m} /\right.$ $\left.\Omega_{c} \mathrm{l}\right)^{1 / 3} \leq 1$, one finds $B(\lambda) / B_{0} \sim\left(\sin \alpha_{R} / \sin \alpha_{0}\right)^{2} \sim\left(p \varepsilon_{m 0} /\right.$ In $\left.+\gamma \omega_{m} / \Omega_{c} \mid\right)^{2 / 3}$ from equation (A1), with $B(\lambda)$ and $B_{0}$ the local and equatorial magnetic field amplitudes. We have then roughly $\sin \alpha_{R} / \sin \alpha_{0}<\left(p \varepsilon_{m 0}\right)^{1 / 3}$ at resonance for $n \neq-1$. $\alpha_{L C}$ is smaller than $5^{\circ}$ for $L>4$ in the outer belt and $p \varepsilon_{m 0}<100$ for $E \leq 3 \mathrm{MeV}$ and $L<7$ for typical density profiles [Sheeley et al., 2001]. Moreover, $\omega_{m} / \Omega_{c 0} \leq 0.35$ typically for lower-band chorus [Agapitov et al., 2011; Shprits et al., 2007; Horne et al., 2005], so that for $E \leq 3 \mathrm{MeV}$ at least, one obtains $\sin \alpha_{R}<\left(p \varepsilon_{m 0}\right)^{1 / 3} \sin \alpha_{L C}<0.5$. Consequently, the resonant value of $\theta$ at the Bessel function maximum, given by $\theta_{R, M}=\pi / 2-\alpha_{R}$, is larger than $\pi / 3$ for $n \neq-1$. This means that only the first cyclotron resonance $n=-1$ provides significant pitch angle diffusion near the loss cone edge. In such a case, the $\mathrm{J}_{0}$ term is indeed dominant in $\Phi_{-1}^{2}$. As concerns the $n=+1$ resonance, it is negligible at small $\theta<\pi / 3$ as compared to the $n=-1$ one [Mourenas and Ripoll, 2012]. Since $\mathrm{J}_{0}(x)$ has a maximum $\mathrm{J}_{0}(x) \sim 1$ for $x<1$, most of the contribution then comes from $x<x_{M}$, i.e. from $\theta<\pi / 2-\alpha_{R}$ and thus from $D^{-}$.

[55] In the intermediate domain $\pi / 3<\theta<\theta_{g}(\lambda)$, some other resonances might contribute for $\cos \theta_{g}<\cos \theta_{R}<$ $\left(p \varepsilon_{m 0}\right)^{1 / 3} \sin \alpha_{L C}$. For typical lower-band chorus frequencies $\omega \geq 0.2 \Omega_{c 0}$ [Agapitov et al., 2011], it requires $p>$ $2\left(\omega / \omega_{c 0}\right)^{1 / 2} \Omega_{c 0} /\left(\omega_{p e} \sin \alpha_{L C}\right)>2.4$, corresponding to high energies $E>1 \mathrm{MeV}$ only. And even at such high energies, equations (A3)-(B2) imply that $\Delta_{n} / \Gamma$ decreases like $1 / n^{2}$. This leads to additional contributions smaller than for $n=-1$ in general, especially for $g_{s}\left(\theta_{g}\right) \ll g_{s}(0)$ at low to moderate latitudes.

[56] Let us turn now to the complementary domain $\theta>\theta_{\text {Min }}^{l} \geq \max \left(\theta_{g}, \pi / 4\right)$. We shall consider only moderate latitudes $<40^{\circ}$ corresponding to equatorial pitch angles larger than $15^{\circ}-20^{\circ}$. Then, the main contributions to diffusion mainly come from the first maximum of the Bessel functions so that equation (B1) applies. From equation (6), it looks like $N_{r}$ might go to $+\infty$ at the resonance angle. However, we shall see below that $\theta$ actually never reaches $\theta_{r}$, so that the actual number of the contributing resonances depends on how fast the $D_{n}^{\alpha \alpha}$ coefficients decrease with $n$. To derive analytical expressions for these coefficients, bounds of integration are needed. These bounds for resonance can be obtained from equation (A1) rewritten as a function of $\theta$ only:

$$
\begin{aligned}
& \cos \theta_{R}=\eta^{-1}\left(1-\frac{\omega_{m}}{\Omega_{c} \cos \theta_{R}}\right) \\
& \eta=\left(p \varepsilon_{m} \cos \alpha_{R} /\left|n+\frac{\gamma \omega_{m}}{\Omega_{c}}\right|\right)^{2}
\end{aligned}
$$

which is a second-order equation in $\cos \theta_{R}$. Real solutions exist only for positive discriminant, which is equivalent to $\eta \leq \Omega_{c} /\left(4 \omega_{m}\right)$. The solution $\cos \theta_{R}$ smaller than $\cos \theta_{g}$ reads as

$$
\cos \theta_{R}=\frac{1-\sqrt{1-4 \frac{\omega_{m} \eta}{\Omega_{c}}}}{2 \eta} \approx \frac{\omega_{m}}{\Omega_{c}}\left(1+\frac{\omega_{m} \eta}{\Omega_{c}}+2\left(\frac{\omega_{m} \eta}{\Omega_{c}}\right)^{2}\right)
$$

from which it can be shown that the resonant angle $\theta_{R}$ is comprised between the Gendrin angle $\theta_{g}$ and the resonance angle $\theta_{r}$, being always strictly smaller than the latter since $\eta$ is finite for finite frequency at a finite distance from mirror points. Actually keeping the ion terms in the dispersion relation would lead to a minimum frequency equal to the lower-hybrid frequency [Smith and Brice, 1964]. It follows immediately that

$$
0<1-\frac{\omega_{m}}{\Omega_{c} \cos \theta_{R}} \approx \frac{\eta \frac{\omega_{m}}{\Omega_{c}}+2\left(\eta \frac{\omega_{m}}{\Omega_{c}}\right)^{2}}{1+\eta \frac{\omega_{m}}{\Omega_{c}}+2\left(\eta \frac{\omega_{m}}{\Omega_{c}}\right)^{2}} \leq \frac{1}{2}
$$

where the value $1 / 2$ is obtained for $\theta_{R}=\theta_{g}$. The estimate equation (B5) stands as a good approximation, since it always remains within less than $45 \%$ of the exact value. Now, since $\theta_{R}<\theta_{r}$, significant diffusion occurs only for $\alpha_{R}>\pi / 2-\theta_{r}$ (except for $|n|=1$ ) and $\alpha_{R} \leq \pi / 2-\theta_{g}$. It follows from equation (B1) at the Bessel function's maximum that diffusion is important at low to moderate latitudes only for

$$
\frac{\omega_{m}}{\Omega_{c}}<\sin \alpha_{R, M} \leq \frac{2 \omega_{m}}{\Omega_{c}}, \text { for }|n|>1
$$


[57] Rewriting equation (B6) with $\left(\sin \alpha_{R, M} / \sin \alpha_{0}\right)^{2}=$ $\Omega_{c} / \Omega_{c 0}$ from adiabatic invariance, one gets

$$
\left(\frac{\omega_{m}}{\Omega_{c 0} \sin \alpha_{0}}\right)^{2 / 3}<\frac{\Omega_{c}}{\Omega_{c 0}} \leq\left(\frac{2 \omega_{m}}{\Omega_{c 0} \sin \alpha_{0}}\right)^{2 / 3} \text {, for }|n|>1
$$

[58] Equations (B7), (A3), and (B1) imply that for $\sin \alpha_{0}>\sin \alpha_{0, M}\left(\theta_{g}\right)=2 \omega_{m} / \Omega_{c 0}$, one has $\alpha_{R} \geq \alpha_{0}>$ $\alpha_{R, M}\left(\theta_{g}\right)>\alpha_{R, M}(\theta)$, equivalent to $x>x_{M}$. From equation (B2), $\Delta_{n}$ should therefore decrease like $x_{M} / x=\tan \alpha_{R, M} / \tan \alpha_{R}$ at larger equatorial pitch angles. The largest contribution to diffusion corresponds to the largest $\theta$-integration range, going from $\theta_{g}$ to $\theta_{r}$. Since $\theta_{R}>\theta_{g}$, taking $\theta_{R} \sim \theta_{g}$ therefore defines a point of maximum contribution. The location of the maximum of each $D_{n}^{\alpha \alpha}$ may then be estimated as the value of $\alpha_{0, R}$ for the $n$th resonance, with equation (A1) taken for $\theta_{g}$ at the equator. It is valid for $\alpha_{0, R}>\alpha_{0, M}\left(\theta_{r}\right)$. For $\alpha_{0}$ larger than both $\alpha_{0, R}$ and $\alpha_{0, M}\left(\theta_{g}\right)$, the peak diffusion value must decrease at least like $1 / \tan \alpha_{R}$ as the $\theta$-integration range decreases $\left(\theta_{R}>\theta_{g}\right.$ then tends toward $\theta_{r}$ to fulfil equation (A1), with $\eta$ going down). Actually, bounceintegration only broadens the maxima reached at the equator when $x>x_{M}$, as already noticed in Mourenas and Ripoll [2012]. This gives an upper-bound on equatorial pitch angle, above which diffusion coefficient $D \sim D^{+}$decreases:

$$
\sin \alpha_{0}^{\operatorname{Max}} \approx \operatorname{Max}\left[\frac{2 \omega_{m}}{\Omega_{c 0}}, \sin \alpha_{\mathrm{P}}\right]
$$

where

$$
\cos \alpha_{\mathrm{P}}=\sqrt{\frac{\Omega_{c 0}}{4 \omega_{U}}}\left|\frac{\max \left(0 ;|n|-\frac{\gamma \omega_{U}}{\Omega_{c 0}}\right)}{p \varepsilon_{U 0}}\right| .
$$

[59] At equatorial pitch angles larger than the limit given by equation (B8), $D_{n}^{\alpha \alpha}$ actually decreases faster than $1 / \tan \alpha_{0}$, because $\alpha_{R} \geq \alpha_{0}$.

[60] On the other hand, $\theta_{g}<\theta_{r}$ defines the upper-bound of resonant $\alpha_{R}$. Therefore, if $\alpha_{R}\left(\theta_{g}\right)$ given by the resonance condition (A1) becomes smaller than $\alpha_{R, M}\left(\theta_{r}\right)$ given by the first inequality in equation(B7), then for $|n|>1$ one gets $x<x_{M}$ and $D^{+}$should decrease very quickly as equatorial pitch angle decreases. This happens for

$$
\max \left[1,\left(\frac{4 \omega_{m}}{\Omega_{c 0}}\right)^{1 / 4} \sqrt{\frac{\cos \alpha_{R}\left(\theta_{g}\right) p \varepsilon_{m 0}}{\left|n+\frac{\gamma \omega_{m} \mid}{\Omega_{c 0}}\right|}}\right]<\max \left[1,\left(\frac{\omega_{m}}{\Omega_{c 0} \sin \alpha_{0}}\right)^{2 / 3}\right]
$$

which for $|n| \geq 2$ and not too high energy (such that $\gamma \omega_{m} /$ $\left.|n| \Omega_{c} \ll 1\right)$ leads to

$$
\sin \alpha_{0}<\sin \alpha_{0}^{\operatorname{Min}}=\min \left[\frac{\omega_{m}}{\Omega_{c 0}},\left(\frac{\omega_{m}}{\Omega_{c 0}}\right)^{5 / 8}\left(\frac{|n|}{2 p \varepsilon_{m 0}}\right)^{3 / 4}\right] .
$$

[61] In the domain defined by (B10), it is the $D^{-}$part of $D$ which becomes dominant. It is evaluated in Appendix $\mathrm{C}$ in the small- $\theta$ range where $\langle D\rangle_{B} \tan \alpha_{0}$ is small at small $\alpha_{0}$ and therefore of major importance for lifetime estimates [Albert and Shprits, 2009]. Conversely, it is neglected in lifetime calculations when the large- $\theta$ part of $g(\theta)$ is included, because $\left(\langle D\rangle_{B} \tan \alpha_{0}\right)^{-1}$ is then always small in the full numerical calculations for the considered parameter range $\Omega_{p e} / \Omega_{c 0}>3, L>4$, and $E>100 \mathrm{keV}$ (see Figure 7).

\section{Appendix C: Small- $\theta$ Part $g_{s}(\theta)$ Contribution of Cyclotron Resonances}

[62] Making use of the very convenient weighted-average re-formulation of the diffusion coefficient by Albert [2007], one has

$$
D_{n}^{\alpha \alpha} \approx \frac{\int_{\theta_{\min }}^{\theta_{\max }} \sin \theta g(\theta) \Delta_{n} G_{\omega} d \theta}{\int_{\theta_{\min }}^{\theta_{\max }} \sin \theta g(\theta) \Gamma d \theta}=\left\langle\frac{\Delta_{n} G_{\omega}}{\Gamma}\right\rangle_{\theta}
$$

with a weighting function $\Gamma \sin \theta g(\theta)$. Let us treat here the small- $\theta$ part of $g(\theta)$, such that $\theta_{\text {Max }}^{s}<\min \left(\pi / 4, \theta_{g}\right)$, allowing us to take $\cos \theta \sim 1$ in general. Such small- $\theta$ waves are actually predominant at low latitudes [Burton and Holzer, 1974; Agapitov et al., 2011; Li et al., 2011]. Then, $\Gamma \propto 1 /$ $\left(\omega_{m} \cos \theta\right)^{3}$, and we have $\Gamma \sin \theta d \theta \sim \Gamma_{\theta=0} \tan \theta / \cos ^{2} \theta d \theta=$ $\Gamma_{\theta=0} y d y$ with the new variable $y=\tan \theta$. As discussed in Appendix B, for small to moderate equatorial pitch angles $\left(\alpha_{0} \leq \pi / 2-\theta_{\text {Max }}^{s} \sim 45^{\circ}\right.$ to $60^{\circ}$, where $D^{+}$is also small [Mourenas and Ripoll, 2012]), we just need to evaluate the $n=-1$ resonance's contribution. Moreover, taking $\left(\mathrm{J}_{0}(1+\cos \theta)+\mathrm{J}_{-2}(1-\cos \theta)\right)^{2} \sim\left(2 \mathrm{~J}_{0}\right)^{2}$ is then a reliable first-order approximation. Neglecting the phase terms, it is reasonable to assume that the Bessel function is nearly constant $\left(\mathrm{J}_{0}(x) \sim 1\right)$ for $x \sim \tan \alpha_{R} \tan \theta \sim 0$ to 1 , for $\theta<\theta_{\mathrm{Max}}^{s} \leq \pi / 3$ and $\alpha_{R}$ lying between $3^{\circ}$ and $25^{\circ}$, not too far from the loss cone. As shown in Appendix B, only the $x<x_{M}$ part is actually contributing. This allows us to write

$$
\frac{\Delta_{-1} G_{\omega}}{\Gamma} \approx \frac{\frac{\pi}{2}\left|1-\frac{\omega_{m}}{\Omega_{c}}\right| G_{\omega}}{\left|1-\frac{2 \omega_{m}}{\Omega_{c} \cos \theta}+\frac{\Omega_{c}}{\gamma \omega_{m}}\right|\left(1-\frac{\gamma \omega_{m}}{\Omega_{c}}\right)^{2}}
$$

which is nearly a constant as a function of $\theta$ (it is justified for $\theta<\pi / 3$ and $\Delta \omega<\omega_{m} / 2$ : see the discussion at the start of section 2.2 and Mourenas and Ripoll [2012]). The term $\Delta_{-1} G_{1} / \Gamma$ can therefore be taken outside of the integral in equation (C1). One obtains the remarkable result that the $\theta$-integrals at the numerator and denominator of equation $(\mathrm{C} 1)$ cancel each other. This means that the precise shape of $g(\theta)$ is unimportant there. One may use a Gaussian or a step-function, the final result will be the same, essentially equivalent to taking $\theta \sim 0$ as in the PPA scheme [Summers, 2005].

[63] This leads to the following expression at the equator for the principal cyclotron harmonic diffusion coefficient near the loss cone edge:

$$
D_{-1} \approx\left(\frac{\Omega_{c} B_{\text {wave }}^{2}}{\gamma^{2} B^{2}}\right) \frac{\pi^{3 / 2} \operatorname{erf}(1) G_{\omega}\left(\omega_{m}\right)\left|1-\frac{\omega_{m}}{\Omega_{c}}\right|}{4\left|1-\frac{2 \omega_{m}}{\Omega_{c}}+\frac{\Omega_{c}}{\gamma \omega_{m}}\right|\left(1-\frac{\gamma \omega_{m}}{\Omega_{c}}\right)^{2}} .
$$


[64] One singularity might occur in (C3) for $\gamma \omega_{m} / \Omega_{c}=1$. Nevertheless, the constraint of the resonance relation (A1) must be taken into account to find the latitude at which resonance eventually occurs, which is equivalent to finding $\Omega_{c 0} / \Omega_{c}$. Numerically solving (iteratively) for the solution in a dipolar geomagnetic field with a typical trough density on the dayside [Sheeley et al., 2001] for $L>4, E<8 \mathrm{MeV}$, and $\theta<\pi / 3$, it appears that $\gamma \omega_{m} / \Omega_{c}<1 / 3$ for $\omega_{m} / \Omega_{c 0}<1 / 3$. It remains true for other resonances $n<1$. Thus, the term $1 /\left(1+\gamma \omega_{m} / n \Omega_{c}\right)$ does not lead to a singularity.

[65] In equation (C3) we take $G_{\omega}\left(\omega_{m}\right)=$ $\Omega_{c} B_{s}^{2}\left(\omega_{m}\right) / \int_{\omega_{L}}^{\omega_{U}} B_{s}^{2}\left(\omega^{\prime}\right) d \omega^{\prime} \approx \Omega_{c} /(\sqrt{\pi} \Delta \omega \operatorname{erf}(1))$ and $\left\langle G_{\omega}\right\rangle_{\omega} \sim$ $\left(\pi^{1 / 2} / 2\right) \operatorname{erf}(1) G_{\omega}\left(\omega_{m}\right)$. Taking directly $\theta=0$ gives in this case essentially the same result as the weighted-average over $\theta=0$ to $\Delta \theta$ as already noticed by Shprits et al. [2006], Albert [2007], and Mourenas and Ripoll [2012]. One can check also that equation (20) from Mourenas and Ripoll [2012] is recovered from equation (C3) in the limit $\left(p \varepsilon_{m 0}\right)^{2 / 3} \Omega_{c 0}$ $\left(\gamma \omega_{m}\right) \gg 1-2 \omega_{m} / \Omega_{c}$.

[66] Provided that $p \varepsilon_{m 0}$ is not too small, as it is usually the case, the contribution to the bounce averaged integral (9) is negligible except over only a small $\lambda$-domain around resonant latitude $\lambda_{R}$. The bounce-integral can therefore be approximated by $\langle D\rangle_{B} \approx D\left(\lambda_{R}\right)\left(\lambda_{\max }-\lambda_{\min }\right) \cos \alpha_{R} \cos ^{7} \lambda_{R} /$ $\left(T\left(\alpha_{0}\right) \cos ^{2} \alpha_{0}\right)$. The domain $\left(\lambda_{\max }-\lambda_{\min }\right)$ is approximated by $\lambda_{\text {max,min }} \approx \lambda_{R} \pm \delta \lambda_{R}$. Using equation (A1), the resonant latitude $\lambda_{R}$ is given by $\cos \lambda_{R} /\left(1+3 \sin ^{2} \lambda_{R}\right)^{1 / 12} \sim\left(\sin \alpha_{0} /\right.$ $\left.\sin \alpha_{R}\right)^{1 / 3} \sim\left(\mid 1-\gamma \omega_{m} / \Omega_{c} l / p \varepsilon_{m 0} \cos \alpha_{R}\right)^{1 / 9} . \delta \lambda_{R}$ is obtained from the same expression evaluated at $\omega_{m}+\Delta \omega$. Series expansions near the first cyclotron resonance assuming small $\lambda$, small $\Delta \omega / \omega_{m}$, and moderate $\alpha_{R}$, finally give $\lambda_{\max }-\lambda_{\min } \approx$ $2 \delta \lambda_{R} \approx \Delta \lambda_{R} \approx 2\left(\sqrt{\lambda_{R}^{2}+2 \Delta \omega /\left(27 \omega_{m}\right) /\left(p \varepsilon_{m 0}\right)^{1 / 9}}-\lambda_{R}\right)$ where we have $\lambda_{R} \approx\left(2 / 3^{1 / 2}\right) \sqrt{1-\left(\left|1-\gamma \omega_{m} / \Omega_{c}\right| / p \varepsilon_{m 0}\right)^{1 / 9}}$. It leads to a first-order estimate of the diffusion coefficient near the loss cone edge:

$$
\begin{aligned}
\left\langle D_{-1}^{\alpha \alpha}\right\rangle_{B}^{s}\left(\alpha_{L C}\right) \approx & \frac{\pi^{3 / 2} \operatorname{erf}(1) B_{w a v e}^{2} \omega_{m} G_{w}\left(\omega_{m}\right)}{4 \gamma B_{0}^{2}\left(p \varepsilon_{m 0}\right)^{13 / 9} T\left(\alpha_{L C}\right)} \times \\
& \times \frac{\Delta \lambda_{R}\left(1+3 \sin ^{2} \lambda_{R}\right)^{7 / 12}\left(1-\frac{\omega_{m}}{\Omega_{c}}\right)}{\left|\frac{\gamma \omega_{m}}{\Omega_{c}}-\frac{2 \gamma \omega_{m}^{2}}{\Omega_{c}^{2}}+1\right|\left|1-\frac{\gamma \omega_{m}}{\Omega_{c}}\right|^{4 / 9}}
\end{aligned}
$$

where $\Omega_{c} / \Omega_{c 0} \approx\left(p \varepsilon_{m 0}\right)^{2 / 3}$ is generally a good approximation.

\section{Appendix D: Large- $\theta$ Part $g_{l}(\theta)$ Contribution of Cyclotron Resonances}

[67] Since $\Gamma \propto\left(\omega_{m} \cos \theta\right)^{-3}\left|1-\omega_{m} /\left(\Omega_{c} \cos \theta\right)\right|^{-1}$ we have $\Gamma \sin \theta d \theta \approx \Gamma_{\theta=0} \tan \theta /\left(\cos ^{2} \theta\left|1-\omega_{m} /\left(\Omega_{c} \cos \theta\right)\right|\right) d \theta=\Gamma_{\theta=0} y d y /$ $\left(1-\omega_{m} /\left(\Omega_{c} \cos \theta\right) \mid\right.$ with the new variable $y=\tan \theta$. Using equation (A1) to replace the term $\left|1-\omega_{m} /\left(\Omega_{c} \cos \theta\right)\right|$ by a term proportional to $\cos \theta=\left(1+y^{2}\right)^{-1 / 2}, D_{ \pm n}^{\alpha \alpha}$ can then be analytically integrated with respect to $y$, provided the Bessel function is conveniently approximated.

[68] Now, we integrate from $\theta_{S 2}$ to $\theta_{\operatorname{Max} 2}$ (see scheme in Figure 3). Let us examine first the singularity in equation (A3) occurring at $1-\omega_{m} /\left(\Omega_{c} \cos \theta\right)=0$. The latter expression must actually be taken at its value for $\left(\theta_{R}, \omega_{R}\left(\theta_{R}\right)\right)$ satisfying the resonance condition (A1). This additional constraint allows substituting the value of $1-\omega_{m} /\left(\Omega_{c} \cos \theta\right)$ by its finite expression given by equation (A1). Now, the $\Phi_{n}$ term can be approximated using $\left((. .) \mathrm{J}_{ \pm n+1}+(. .) \mathrm{J}_{ \pm n-1}\right)^{2} \approx\left( \pm 1-\omega_{m} /\right.$ $\left.\Omega_{c}\right)^{2} \mathrm{~J}_{|n|-1}^{2}$ at its maximum value for $\theta \sim \theta_{g}$, since the largest Bessel function is that of the smallest index. Further using equation (B2), the integration of $\Delta_{n} G_{\omega}$ with respect to $\theta$ in equation $(\mathrm{C} 1)$ can then be carried out with the assumption that the term $\left|1-2 \omega_{m} /\left(\Omega_{c} \cos \theta\right)-n \Omega_{c} /\left(\gamma \omega_{m}\right)\right|$ is only weakly dependent on $\theta$, actually taking it at $\theta_{g}$ since $\theta_{g} \leq \theta_{R}$. With the variable $y=\tan \theta$, it is equivalent to integrating $1+y^{2}$ in the numerator of equation $(\mathrm{C} 1)$ with respect to $y$. This leads to

$$
\begin{aligned}
\left\langle D_{ \pm n}^{+}\right\rangle_{\theta_{S 2}}^{\theta_{\text {Max } 2} \approx} & \frac{\sqrt{\pi} \operatorname{erf}(1) B_{\text {wave }}^{2} \Omega_{c} G_{\omega}\left(\omega_{m}\right) \ln \mid\left(1 \mp \frac{\omega_{m}}{\Omega_{c}}\right)^{2}}{8 B^{2}\left|\frac{n \Omega_{c}}{\gamma \omega_{m}}\right|\left(\gamma p \varepsilon_{m} \cos \alpha\right)^{2} \tan \alpha} \times \\
& \times \frac{\tan \theta_{\operatorname{Max} 2}\left(3+\tan ^{2} \theta_{\text {Max } 2}\right)-\tan \theta_{S 2}\left(3+\tan ^{2} \theta_{S 2}\right)}{\left(1+\tan ^{2} \theta_{\text {Max }}^{l}\right)^{3 / 2}-\left(1+\tan ^{2} \theta_{\text {Min }}^{l}\right)^{3 / 2}}
\end{aligned}
$$

[69] The diffusion coefficient (D1) can be large at moderate to large pitch angles given by equation (8), since the $n$-resonances levels in equation (D1) are nearly independent of $n$, so that many resonances can contribute. Nevertheless, pitch angle $\alpha_{R}$ is limited by condition (A1) with cos $\alpha_{R}<\sin \theta_{r}$, showing that $\theta_{R}\left(\lambda_{R}\right)$ can take any value smaller than $\theta_{r}\left(\lambda_{R}\right)$ only if $|n|<N_{r}\left(\theta_{g 0}\right)$ given in equation (7). At larger $n$-values, $\theta_{R}\left(\lambda_{R}\right)$ tends toward $\theta_{r}\left(\lambda_{R}\right)$ so that the integration range $\cos \theta_{R}\left(\lambda_{R}\right)-\cos \theta_{r}\left(\lambda_{R}\right) \approx \eta \propto 1 / n^{2}$ from equation (B3), leading in equation (D1) to $D_{n}^{\alpha \alpha} \propto 1 / n^{2}$ at large $n$, shrinking quickly at higher $|n|$ and/or $\Omega_{c}$ (or higher latitudes). Consequently, the $D^{+}$diffusion coefficient may be rewritten as

$$
\sum D_{n} \approx 2 N_{r}\left(\theta_{g 0}\right)\left(D_{+1}+D_{-1}\right) .
$$

[70] Aside from the $\theta$-bounds, the integrand in equation (9) with equation (D1) is proportional to $\Omega_{c}^{3 / 2} \cos ^{7} \lambda \sim \Omega_{c}^{1 / 2}$, meaning it is only slowly varying with latitude. As concerns the $\theta$-bounds, it is reasonable to expect mean value $\theta_{m}^{l} \approx\left(\theta_{\text {Min }}^{l}+\theta_{\text {Max }}^{l}\right) / 2$ to increase as the waves propagate away from the equator, varying roughly like (or slightly more than) the Gendrin angle, leading to $1 / \tan \theta_{m}^{l} \propto \cos \theta_{g} \propto$ $\omega_{m} / \Omega_{c}$. Dayside chorus waves observations seem to confirm this, showing in addition that variance $\sim \theta_{\text {Max }}^{l}-\theta_{\text {Min }}^{l}$ also increases in a similar fashion with latitude [Agapitov et al., 2011; Burton and Holzer, 1974; Artemyev et al., 2012]. Assuming for simplicity that the bounds of integration $\theta_{\mathrm{Max} 2} \sim \theta_{r}$ and $\theta_{S 2} \propto \theta_{g}$ vary similarly too, the total variation of the integrand is weaker than $\Omega_{c}^{1 / 2}$. Since $\lambda_{\max }$ is small, the bounce-integral (9) can then be approximated by the value of its integrant at $\lambda_{R} \sim 0$ multiplied by the domain $\lambda_{\max }-\lambda_{\min }$. It leads to a first-order estimate:

$$
\begin{aligned}
\left\langle D_{ \pm n}^{+}\right\rangle_{B}^{l}\left(\alpha_{0}\right) \approx & \frac{\sqrt{\pi} \operatorname{erf}(1) B_{w}^{2} \omega_{m} G_{\omega}\left(\omega_{m}\right) \lambda_{\max }\left(1+\frac{\omega_{m}^{2}}{\Omega_{c 0}}\right)}{8 \gamma B_{0}^{2} T\left(\alpha_{0}\right) \cos ^{2} \alpha_{0} \sin \alpha_{0}\left(p \varepsilon_{m 0}\right)^{2}} \times \\
& \times \frac{\tan \theta_{\text {Max } 2}\left(3+\tan ^{2} \theta_{\text {Max } 2}\right)-\tan \theta_{S 2}\left(3+\tan ^{2} \theta_{S 2}\right)}{\left(1+\tan ^{2} \theta_{\text {Max }}^{l}\right)^{3 / 2}-\left(1+\tan ^{2} \theta_{\text {Min }}^{l}\right)^{3 / 2}}
\end{aligned}
$$


[71] In equation (D2), the $\theta$-bounds are evaluated at $\lambda_{R}=0$. The magnitude of large- $\theta D_{ \pm n}^{+}$is almost independent of $n$ in equation (D2), so that the contributions of many resonances can lead to large increase in diffusion in the domain of moderate to large equatorial pitch angles $\alpha_{0}>\alpha_{0}^{\text {Min }}$, with $\alpha_{0}^{\text {Min }}$ given by equation (B10). This is indeed confirmed by full numerical calculations of the diffusion coefficients (see Figures 2 and 8 and Artemyev et al. [2012]). The lower-part of integration from $\theta_{\text {Min }}^{l}$ to $\theta_{S 1}$, has yet to be evaluated. But the latter term, which leads to integration of $\mathrm{J}_{|n|-1}^{2}(y) y\left(1+y^{2}\right)$ in the numerator of equation (C1), is negligible by construction as compared to $D^{+}$at moderate to large pitch angles. The small- $\theta$ part of $D^{+}$, which becomes important above $60^{\circ}$ has already been estimated in equations (30) and (34) of Mourenas and Ripoll [2012], which remain approximately valid here when $\left(p \varepsilon_{m 0}\right)^{2 / 3} \Omega_{c 0} /\left(\gamma \omega_{m}\right) \gg\left|1-2 \omega_{m} / \Omega_{c}\right|$

\section{Appendix E: Numerical Calculation of the Diffusion Coefficients and Lifetimes}

[72] In this paper we use the scheme of calculation of the diffusion coefficients (1) described by Glauert and Horne [2005]. However, we also take into account significant improvements for distribution $g(\theta)$ based on CLUSTER statistics of whistler wave propagations [Agapitov et al., 2011]. We use a Gaussian function $g(\theta)=\exp (-(\tan \theta-$ $\left.\tan \theta_{m}\right)^{2} / \tan ^{2} \Delta \theta$ ) with $\tan \theta_{m}$ and $\tan \Delta \theta$ depending on the magnetic latitude $\lambda$. These dependencies are obtained from CLUSTER statistics and approximated by polynomials of $\lambda$ [see details in Artemyev et al., 2012]. The calculation of local pitch angle diffusion coefficient $D_{n}^{\alpha \alpha}$ for given $\lambda$, electron energy and pitch angle $\alpha$ consists in the following steps:

[73] 1 . For each value of $\theta \in\left[\theta_{\min }, \theta_{\max }\right]$ we solve the system consisting of the resonance condition (5) and dispersion relation $\omega=\Omega_{c} \cos \theta /\left(1+\Omega_{p e}^{2} /(k c)^{2}\right)$ to obtain resonant frequency $\omega_{i, n}$ and corresponding wave number $k_{i, n}$. Here $n$ is the order of cyclotron harmonic resonance and $i$ is the index of the resonant root.

[74] 2. For all obtained sets $\left\{\omega_{i, n}, k_{i, n}\right\}$ we calculate the corresponding power density $G_{\omega}\left(\omega_{i, n}, k_{i, n}\right)$ according to equation (3).

[75] 3. Then we integrate over $\theta$ with function $G_{\theta}$, where $g(\theta)=\exp \left(-\left(\tan \theta-\tan \theta_{m}\right)^{2} / \tan ^{2} \Delta \theta\right)$ is obtained for a given $\lambda$.

[76] As a result, we obtain $D_{n}^{\alpha \alpha}$ depending on $\lambda, \alpha$ and electron energy. Then we sum all cyclotron harmonics and integrate over bounce oscillations according to equation (9), recalculating pitch angle by using conservation of the magnetic moment:

$$
\sin ^{2} \alpha=\sin ^{2} \alpha_{e q} b(\lambda)
$$

where $b(\lambda)=\sqrt{1+3 \sin ^{2} \lambda} / \cos ^{6} \lambda$. We average over bounce oscillations up to $\lambda=\min \left(\lambda_{\text {mirror }}, 40^{\circ}\right)$, where $\lambda_{\text {mirror }}$ corresponds to mirror points, i.e. $\sin ^{2} \alpha_{e q} b\left(\lambda_{\text {mirror }}\right)=1$. This gives the final diffusion coefficients $\langle D\rangle$. We also use these pitch angle diffusion coefficients $\langle D\rangle$ to calculate lifetimes according to Albert and Shprits [2009].

[77] In some cases, we also calculate diffusion coefficients with constant parameters $\tan \theta_{m}=0, \tan \Delta \theta=0.577$ with $\theta_{\text {Max }}=45^{\circ}$. These parameters are the same as used by Glauert and Horne [2005] and Horne et al. [2005]. Results obtained for these parameters are named in the paper as calculation without large- $\theta$ part.

[78] According to CLUSTER observations the mean value $\tan \theta_{m}$ as well as the variance $\tan \Delta \theta$ grow with magnetic latitude [Agapitov et al., 2011]. As a result, for moderate to large values of $\lambda$ one has significantly oblique direction of wave propagation $\left(\tan \theta_{m} \gg 1\right)$ and distribution $g(\theta)$ is more dispersive (i.e. $\tan \Delta \theta \gg 1$ ). This effect leads to the intensification of higher order cyclotron harmonics [see, e.g., Shklyar and Matsumoto, 2009] and results in an increase of pitch angle diffusion [Shprits and Ni, 2009; Artemyev et al., 2012].

[79] Acknowledgments. The work of A.V.O. was supported by the STUDIUM program of the Region Centre in France. The work of A.V.A. was supported by the grant CNES Modele d'ondes.

[80] Robert Lysak thanks the reviewers for their assistance in evaluating this paper

\section{References}

Abramowitz, M., and I. A. Stegun (1972), Handbook of Mathematical Functions, Dover, New York.

Agapitov, O., V. Krasnoselskikh, Y. Zaliznyak, V. Angelopoulos, O. Le Contel, and G. Rolland (2010), Chorus source region localization in the Earth's outer magnetosphere using THEMIS measurements, Ann. Geophys., 28, 1377-1386.

Agapitov, O., V. Krasnoselskikh, Y. V. Khotyaintsev, and G. Rolland (2011), A statistical study of the propagation characteristics of whistler waves observed by Cluster, Geophys. Res. Lett., 38, L20103, doi:10.1029/ 2011 GL049597.

Albert, J. M. (2005), Evaluation of quasi-linear diffusion coefficients for whistler mode waves in a plasma with arbitrary density ratio, J. Geophys. Res., 110, A03218, doi:10.1029/2004JA010844.

Albert, J. M. (2007), Simple approximations of quasi-linear diffusion coefficients, J. Geophys. Res., 112, A12202, doi:10.1029/2007JA012551.

Albert, J. M. (2008), Efficient approximations of quasi-linear diffusion coefficients in the radiation belts, J. Geophys. Res., 113, A06208, doi:10.1029/2007JA012936.

Albert, J. M., and Y. Y. Shprits (2009), Estimates of lifetimes against pitch angle diffusion, J. Atmos. Sol. Terr. Phys., 71, 1647-1652.

Andronov, A. A., and V. Y. Trakhtengerts (1964), Kinetic instability of the Earth's outer radiation belt, Geomagn. Aeron., 4, 233-242.

Artemyev, A., O. Agapitov, H. Breuillard, V. Krasnoselskikh, and G. Rolland (2012), Electron pitch-angle diffusion in radiation belts: The effects of whistler wave oblique propagation, Geophys. Res. Lett., 39, L08105, doi:10.1029/2012GL051393.

Barker, A. B., X. Li, and R. S. Selesnick (2005), Modeling the radiation belt electrons with radial diffusion driven by the solar wind, Space Weather, 3, S10003, doi:10.1029/2004SW000118.

Borovsky, J. E., and M. H. Denton (2011), A survey of the anisotropy of the outer electron radiation belt during high-speed-stream-driven storms, J. Geophys. Res., 116, A05201, doi:10.1029/2010JA016151.

Bortnik, J., L. Chen, W. Li, R. M. Thorne, and R. B. Horne (2011), Modeling the evolution of chorus waves into plasmaspheric hiss, J. Geophys. Res., 116, A08221, doi:10.1029/2011JA016499.

Boskova, J., F. Jiricek, P. Triska, B. V. Lundin, and D. R. Shkliar (1990), A possible common nature of equatorial half-gyrofrequency VLF emissions and discrete plasmaspheric emissions, Ann. Geophys., 8, 755-763.

Bourdarie, S., D. Boscher, T. Beutier, J.-A. Sauvaud, and M. Blanc (1996), Magnetic storm modeling in the Earth's electron belt by the Salammbô code, J. Geophys. Res., 101, 27,171-27,176.

Burton, R. K., and R. E. Holzer (1974), The origin and propagation of chorus in the outer magnetosphere, J. Geophys. Res., 79, 1014-1023.

Chum, J., and O. Santolík (2005), Propagation of whistler-mode chorus to low altitudes: divergent ray trajectories and ground accessibility, Ann. Geophys., 23, 3727-3738.

Davidson, G. T. (1976), An improved empirical description of the bounce motion of trapped particles, J. Geophys. Res., 81, 4029-4030.

Davidson, G. T. (1986), Pitch-angle diffusion in morningside aurorae, J. Geophys. Res., 91, 4413-4427. 
Denton, R. E., J. Goldstein, and J. D. Menietti (2002), Field line dependence of magnetospheric electron density, Geophys. Res. Lett., 29(24), 2205, doi:10.1029/2002GL015963.

Edgar, B. C. (1976), The upper- and lower-frequency cutoffs of magnetospherically reflected whistlers, J. Geophys. Res., 81, 205-211.

Fok, M.-C., A. Glocer, Q. Zheng, R. B. Horne, N. P. Meredith, J. M. Albert, and T. Nagai (2011), Recent developments in the radiation belt environment model, J. Atmos. Sol. Terr. Phys., 73, 1435-1443.

Gendrin, R. (1961), Le guidage des whistlers par le champ magnetique, Planet. Space Sci., 5, 274-278.

Glauert, S. A., and R. B. Horne (2005), Calculation of pitch angle and energy diffusion coefficients with the PADIE code, J. Geophys. Res., 110, A04206, doi:10.1029/2004JA010851.

Goldstein, B. E., and B. T. Tsurutani (1984), Wave normal directions of chorus near the equatorial source region, J. Geophys. Res., 89, $2789-2810$

Gu, X., Z. Zhao, B. Ni, Y. Shprits, and C. Zhou (2011), Statistical analysis of pitch angle distribution of radiation belt energetic electrons near the geostationary orbit: CRRES observations, J. Geophys. Res., 116, A01208, doi:10.1029/2010JA016052.

Hamlin, D. A., R. Karplus, R. C. Vik, and K. M. Watson (1961), Mirror and azimuthal drift frequencies for geomagnetically trapped particles, J. Geophys. Res., 66, 1-4.

Haque, N., M. Spasojevic, O. Santolík, and U. S. Inan (2010), Wave normal angles of magnetospheric chorus emissions observed on the Polar spacecraft, J. Geophys. Res., 115, A00F07, doi:10.1029/2009JA014717.

Haque, N., U. S. Inan, T. F. Bell, J. S. Pickett, J. G. Trotignon, and G. Facskó (2011), Cluster observations of whistler mode ducts and banded chorus, Geophys. Res. Lett., 38, L18107, doi:10.1029/2011GL049112.

Hattori, K., M. Hayakawa, D. Lagoutte, M. Parrot, and F. Lefeuvre (1991), Further evidence of triggering chorus emissions from wavelets in the hiss band, Planet. Space Sci., 39, 1465-1472.

Hayakawa, M., K. Hattori, S. Shimakura, M. Parrot, and F. Lefeuvre (1990), Direction finding of chorus emissions in the outer magnetosphere and their generation and propagation, Planet. Space Science, 38, 135-137.

Helliwell, R. A. (1965), Whistlers and Related Ionospheric Phenomena, Stanford Univ. Press, Stanford, Calif.

Helliwell, R. A. (1995), The role of the Gendrin mode of VLF propagation in the generation of magnetospheric emissions, Geophys. Res. Lett., 22 2095-2098.

Horne, R. B., R. M. Thorne, S. A. Glauert, J. M. Albert, N. P. Meredith, and R. R. Anderson (2005), Timescale for radiation belt electron acceleration by whistler mode chorus waves, J. Geophys. Res., 110, A03225, doi:10.1029/2004JA010811.

Jirícek, F., D. R. Shklyar, and P. Tríska (2001), LHR effects in nonducted whistler propogation-New observations and numerical modelling, Ann. Geophys., 19, 147-157.

Kennel, C. F., and H. E. Petschek (1966), Limit on stably trapped particle fluxes, J. Geophys. Res., 71, 1-28.

Lauben, D. S., U. S. Inan, T. F. Bell, and D. A. Gurnett (2002), Source characteristics of ELF/VLF chorus, J. Geophys. Res., 107(A12), 1429, doi:10.1029/2000JA003019.

Li, W., Y. Y. Shprits, and R. M. Thorne (2007), Dynamic evolution of energetic outer zone electrons due to wave-particle interactions during storms, J. Geophys. Res., 112, A10220, doi:10.1029/2007JA012368.

Li, W., J. Bortnik, R. M. Thorne, and V. Angelopoulos (2011), Global distribution of wave amplitudes and wave normal angles of chorus waves using THEMIS wave observations, J. Geophys. Res., 116, A12205, doi:10.1029/2011JA017035.

Lyons, L. R. (1973), Comments on "pitch-angle diffusion in the radiation belts, J. Geophys. Res., 78, 6793-6795.

Lyons, L. R. (1974), Pitch angle and energy diffusion coefficients from resonant interactions with ion-cyclotron and whistler waves, J. Plasma Phys., 12, 417-432.

Lyons, L. R., R. M. Thorne, and C. F. Kennel (1971), Electron pitch-angle diffusion driven by oblique whistler-mode turbulence, J. Plasma Phys., 6, 589-606.

Lyons, L. R., R. M. Thorne, and C. F. Kennel (1972), Pitch-angle diffusion of radiation belt electrons within the plasmasphere, J. Geophys. Res., 77, 3455-3474.

Lyons, L. R., D.-Y. Lee, R. M. Thorne, R. B. Horne, and A. J. Smith (2005), Solar wind-magnetosphere coupling leading to relativistic electron energization during high-speed streams, J. Geophys. Res., 110, A11202, doi:10.1029/2005JA011254.

Meredith, N. P., R. B. Horne, and R. R. Anderson (2001), Substorm dependence of chorus amplitudes: Implications for the acceleration of electrons to relativistic energies, J. Geophys. Res., 106, 13,165-13,178.
Meredith, N. P., R. B. Horne, S. A. Glauert, D. N. Baker, S. G. Kanekal, and J. M. Albert (2009), Relativistic electron loss timescales in the slot region, J. Geophys. Res., 114, A03222, doi:10.1029/2008JA013889.

Mourenas, D., and J.-F. Ripoll (2012), Analytical estimates of quasi-linear diffusion coefficients and electron lifetimes in the inner radiation belt J. Geophys. Res., 117, A01204, doi:10.1029/2011JA016985.

Muto, H., M. Hayakawa, M. Parrot, and F. Lefeuvre (1987), Direction finding of half-gyrofrequency VLF emissions in the off-equatorial region of the magnetosphere and their generation and propagation, J. Geophys. Res., 92, 7538-7550.

Ni, B., R. M. Thorne, Y. Y. Shprits, and J. Bortnik (2008), Resonant scattering of plasma sheet electrons by whistler-mode chorus: Contribution to diffuse auroral precipitation, Geophys. Res. Lett., 35, L11106, doi:10.1029/2008GL034032.

Nishimura, Y., et al. (2011), Multievent study of the correlation between pulsating aurora and whistler mode chorus emissions, J. Geophys. Res., 116, A11221, doi:10.1029/2011JA016876

Pokhotelov, D., F. Lefeuvre, R. B. Horne, and N. Cornilleau-Wehrlin (2008), Survey of ELF-VLF plasma waves in outer radiation belt observed by Cluster STAFF-SA experiment, Ann. Geophys., 26, 3269-3277.

Santolík, O., D. A. Gurnett, J. S. Pickett, J. Chum, and N. CornilleauWehrlin (2009), Oblique propagation of whistler mode waves in the chorus source region, J. Geophys. Res., 114, A00F03, doi:10.1029/ 2009JA014586.

Schulz, M. (1974), Particle lifetimes in strong diffusion, Astrophys. Space Sci., 31, 37-42.

Sheeley, B. W., M. B. Moldwin, H. K. Rassoul, and R. R. Anderson (2001), An empirical plasmasphere and trough density model: CRRES observations, J. Geophys. Res., 106, 25,631-25,642.

Shklyar, D. R., and F. Jiř́íček (2000), Simulation of nonducted whistler spectrograms observed aboard the MAGION 4 and 5 satellites, J. Atmos. Sol. Terr. Phys., 62, 347-370.

Shklyar, D., and H. Matsumoto (2009), Oblique whistler-mode waves in the inhomogeneous magnetospheric plasma: Resonant interactions with energetic charged particles, Surv. Geophys., 30, 55-104.

Shprits, Y. Y., and B. Ni (2009), Dependence of the quasi-linear scattering rates on the wave normal distribution of chorus waves, J. Geophys. Res. 114, A11205, doi:10.1029/2009JA014223

Shprits, Y. Y., R. M. Thorne, R. B. Horne, and D. Summers (2006), Bounce-averaged diffusion coefficients for field-aligned chorus waves, J. Geophys. Res., 111, A10225, doi:10.1029/2006JA011725.

Shprits, Y. Y., N. P. Meredith, and R. M. Thorne (2007), Parameterization of radiation belt electron loss timescales due to interactions with chorus waves, Geophys. Res. Lett., 34, L11110, doi:10.1029/2006GL029050.

Shprits, Y. Y., D. A. Subbotin, N. P. Meredith, and S. R. Elkington (2008), Review of modeling of losses and sources of relativistic electrons in the outer radiation belt II: Local acceleration and loss, J. Atmos. Sol. Terr. Phys., 70, 1694-1713.

Smith, R. L., and N. Brice (1964), Propagation in multicomponent plasmas, J. Geophys. Res., 69, 5029-5040.

Summers, D. (2005), Quasi-linear diffusion coefficients for fieldaligned electromagnetic waves with applications to the magnetosphere, J. Geophys. Res., 110, A08213, doi:10.1029/2005JA011159.

Summers, D., B. Ni, and N. P. Meredith (2007a), Timescales for radiation belt electron acceleration and loss due to resonant wave-particle interactions: 1. Theory, J. Geophys. Res., 112, A04206, doi:10.1029/ 2006JA011801.

Summers, D., B. Ni, and N. P. Meredith (2007b), Timescales for radiation belt electron acceleration and loss due to resonant wave-particle interactions: 2. Evaluation for VLF chorus, ELF hiss, and electromagnetic ion cyclotron waves, J. Geophys. Res., 112, A04207, doi:10.1029/ 2006JA011993.

Tao, X., J. Bortnik, J. M. Albert, K. Liu, and R. M. Thorne (2011), Comparison of quasilinear diffusion coefficients for parallel propagating whistler mode waves with test particle simulations, Geophys. Res. Lett., 38, L06105, doi:10.1029/2011GL046787.

Trakhtengerts, V. Y. (1999), A generation mechanism for chorus emission, Ann. Geophys., 17, 95-100.

Tsurutani, B. T., and E. J. Smith (1977), Two types of magnetospheric ELF chorus and their substorm dependences, J. Geophys. Res., 82, 5112-5128.

Tsurutani, B. T., O. P. Verkhoglyadova, G. S. Lakhina, and S. Yagitani (2009), Properties of dayside outer zone chorus during HILDCAA events: Loss of energetic electrons, J. Geophys. Res., 114, A03207, doi:10.1029/2008JA013353. 
Ukhorskiy, A. Y., K. Takahashi, B. J. Anderson, and H. Korth (2005), Impact of toroidal ULF waves on the outer radiation belt electrons, J. Geophys. Res., 110, A10202, doi:10.1029/2005JA011017.

Varotsou, A., D. Boscher, S. Bourdarie, R. B. Horne, N. P. Meredith, S. A Glauert, and R. H. Friedel (2008), Three-dimensional test simulations of the outer radiation belt electron dynamics including electron-chorus resonant interactions, J. Geophys. Res., 113, A12212, doi:10.1029/ 2007JA012862.
Verkhoglyadova, O. P., and B. T. Tsurutani (2008), Polarization properties of Gendrin mode waves observed in the Earth's magnetosphere: Observations and theory, Ann. Geophys., 27, 4429-4433.

Xiao, F., Z. Su, H. Zheng, and S. Wang (2009), Modeling of outer radiation belt electrons by multidimensional diffusion process, J. Geophys. Res., 114, A03201, doi:10.1029/2008JA013580. 